\title{
ON THE CAUCHY PROBLEM FOR REACTION-DIFFUSION EQUATIONS
}

\author{
XUEFENG WANG
}

Abstract. The simplest model of the Cauchy problem considered in this paper is the following

$$
\begin{array}{ll}
u_{t}=\Delta u+u^{p}, & x \in R^{n}, t>0, u \geq 0, p>1, \\
\left.u\right|_{t=0}=\phi \in C_{B}\left(R^{n}\right), & \phi \geq 0, \phi \neq 0 .
\end{array}
$$

It is well known that when $1<p \leq(n+2) / n$, the local solution of $(*)$ blows up in finite time as long as the initial value $\phi$ is nontrivial; and when $p>(n+2) / n$, if $\phi$ is "small", $(*)$ has a global classical solution decaying to zero as $t \rightarrow+\infty$, while if $\phi$ is "large", the local solution blows up in finite time. The main aim of this paper is to obtain optimal conditions on $\phi$ for global existence and to study the asymptotic behavior of those global solutions. In particular, we prove that if $n \geq 3, p>n /(n-2)$,

$$
0 \leq \phi(x) \leq \lambda u_{s}(x)=\lambda\left(\frac{2(n-2)}{(p-1)^{2}}\left(p-\frac{n}{n-2}\right)\right)^{1 /(p-1)}|x|^{-2 /(p-1)}
$$

( $u_{s}$ is a singular equilibrium of $\left.(*)\right)$ where $0<\lambda<1$, then $(*)$ has a (unique) global classical solution $u$ with $0 \leq u \leq \lambda u_{s}$ and

$$
u(x, t) \leq\left(\left(\lambda^{1-p}-1\right)(p-1) t\right)^{-1 /(p-1)} .
$$

(This result implies that $u_{0} \equiv 0$ is stable w.r.t. to a weighted $L^{\infty}$ topology when $n \geq 3$ and $p>n /(n-2)$.) We also obtain some sufficient conditions on $\phi$ for global nonexistence and those conditions, when combined with our global existence result, indicate that for $\phi$ around $u_{s}$, we are in a delicate situation, and when $p$ is fixed, $u_{0} \equiv 0$ is "increasingly stable" as the dimension $n \uparrow+\infty$. A slightly more general version of $(*)$ is also considered and similar results are obtained.

\section{INTRODUCTION}

The simplest model of the Cauchy problem considered in this paper is the following:

$$
\begin{aligned}
& u_{t}=\Delta u+u^{p}, \quad x \in R^{n}, t>0, p>1, \\
& \left.u\right|_{t=0}=\phi \in C_{B}\left(R^{n}\right) \equiv C\left(R^{n}\right) \cap L^{\infty}\left(R^{n}\right), \quad \phi \geq 0, \quad \phi \not \equiv 0 .
\end{aligned}
$$

$(0.1)$ is related to many equations arising from mathematical biology and chemical reactor theory, and the results for $(0.1)$ may be used to the study of those equations as shown by Aronson and Weinberger [1]. Besides the practical interest in it, (0.1) and its various generalizations have been model problems for

Received by the editors August 16, 1990.

1991 Mathematics Subject Classification. Primary 35K57, 35B35, 35B40; Secondary 35 J60. 
the mathematical study of pathological phenomena and have been studied by many authors in the last two decades (see Levine's survey articles [25] and [26]). Yet our understanding of $(0.1)$ is still incomplete. This is partially due to the apparent lack of compactness and the lack of a good Liapunov functional. In this paper, we shall use the equilibria of $(0.1)$, which are comparatively well understood, to study the Cauchy problem $(0.1)$.

Before we turn to the history of $(0.1)$ in the direction concerned in this paper, we first mention the following standard fact. For any $\phi \in C_{B}\left(R^{n}\right)$ with $\phi \geq 0$, there exists $T_{\phi}>0$ such that $(0.1)$ has a unique classical solution $u$ on $R^{n} \times\left[0, T_{\phi}\right)$ such that $u$ is bounded on $R^{n} \times\left[0, T^{\prime}\right]$ for any $0<T^{\prime}<T_{\phi}$, and if $T_{\phi}<+\infty$, then $\|u(\cdot, t)\|_{L^{\infty}\left(R^{n}\right)} \rightarrow+\infty$ as $t \rightarrow T_{\phi}^{-}$. This can be proved by the standard contraction mapping method. When $T_{\phi}<+\infty$, we say that $u$ blows up in finite time; when $T_{\phi}=+\infty$, we say that $u$ is a global solution.

The study of $(0.1)$ goes back to the fundamental work of Fujita [11]. He proved that when $1<p<(n+2) / n$, the local classical solution blows up in finite time (the same is true when $p=(n+2) / n$, as was proved by Hayakawa [19] and later by Kobayashi, Siaro, and Tanaka [22]), and when $p>(n+2) / n$, for any $k>0$, there exists a small $\delta=\delta(p, n, k)>0$ such that when $0 \leq$ $\phi(x) \leq \delta \exp \left(-k|x|^{2}\right)$ on $R^{n},(0.1)$ has a global classical solution decaying like $t^{-n / 2}$ as $t \rightarrow+\infty$. Also, as a consequence of Lemma 2.1 in [11], if the local solution can be extended globally, then necessarily $e^{t \Delta} \phi \leq t^{-\beta} \beta^{\beta}$ where $\beta=1 /(p-1)$ and

$$
e^{t \Delta} \phi=(4 \pi t)^{-n / 2} \int_{R^{n}} \exp \left(-\frac{|x-y|^{2}}{4 t}\right) \phi(y) d y
$$

(Weissler [34] also obtained such a result in the $L^{p}$ setting). From this, one sees that if $\phi$ is "large", then the local solution of $(0.1)$ blows up in finite time even when $p>(n+2) / n$.

In the direction of relaxing Fujita's sufficient condition on the initial value $\phi$ for global existence, Weissler [35] proved that if $\phi$ satisfies

$$
(p-1) \int_{0}^{\infty}\left\|e^{t \Delta} \phi\right\|_{\infty}^{p-1} d t \leq 1,
$$

then (0.1) has a global classical solution which, when the strict inequality holds, goes to zero uniformly on $R^{n}$ as $t \rightarrow+\infty$. One checks easily that when $\phi$ is dominated by a small multiple of $(1+|x|)^{-m}$ for a large $m,(0.2)$ is satisfied.

In a recent paper, Lee and $\mathrm{Ni}$ [24] gave another sufficient condition for global existence which says that when $p>(n+2) / n$, for any $k>0$, there exists a small $b=b(p, n, k)$ such that if $0 \leq \phi(x) \leq b\left(k+|x|^{2}\right)^{-1 /(p-1)}$, then (0.1) has a global classical solution decaying like $t^{-1 /(p-1)}$ as $t \rightarrow+\infty$ (other decay rates were also obtained if $\phi$ decays faster). They also obtained a nice necessary condition on $\phi$ for global existence of classical solutions of $(0.1)$ :

$$
\liminf _{|x| \rightarrow+\infty}|x|^{2 /(p-1)} \phi(x) \leq\left(\lambda\left(B_{1}\right)\right)^{1 /(p-1)}
$$

where $B_{1}$ is the unit ball in $R^{n}$, and $\lambda\left(B_{1}\right)$ is the first eigenvalue of $-\Delta$ with zero Dirichlet boundary condition on $\partial B_{1}$. These results of Lee and $\mathrm{Ni}$ indicate that the slowest decay of $\phi$ at $|x|=+\infty$ permitting global existence for $(0.1)$ is $|x|^{-2 /(p-1)}$. 
The main aim of this paper is to obtain optimal conditions on $\phi$ for existence of global classical solutions of $(0.1)$ (and for a more general version of $(0.1)$, see (0.6) below), and to study the large time behavior of those solutions. For $(0.1)$, in view of the above discussion, we shall pay particular attention to those $\phi$ with "critical decay" (that is, those for which $\phi|x|^{2 /(p-1)}=O(1)$ as $|x| \rightarrow \infty$ ), and we are especially interested in the following question:

(Q) How large can the $b$ in Lee and Ni's sufficient condition be if we want global classical solutions of $(0.1)$ ?

Note that by $(0.3), b \leq\left(\lambda\left(B_{1}\right)\right)^{1 /(p-1)}$. Our partial answer to $(\mathrm{Q})$ is a consequence of the general results in this paper.

As mentioned before, our main results and analyses rely on a good understanding of the equilibria (regular or singular at $x=0$ ) for which the study dates back to the beginning of this century. Before we state our results, we first mention some elements of the theory of the elliptic counterpart of $(0.1)$ :

$$
\Delta u+u^{p}=0, \quad x \in R^{n}, u>0, n \geq 3 .
$$

This equation is called the Lane-Emden equation. It arises in astrophysics and Riemannian geometry. The fundamental work on $(0.4)$ is due to Fowler [6, 7]. Other references include [5, 20, 27, 30], to cite just a few. In Propositions $3.4,3.5$, and 3.7 we recall or prove some results about $(0.4)$ (and the generalized version of $(0.4))$. At this point, rather than presenting the whole list, we mention that

$\left(\mathrm{E}_{1}\right)$ When $p>n /(n-2), u_{s}(r)=L r^{-2 /(p-1)}(r=|x|)$ is a singular solution of $(0.4))$, where

$$
L=\left(\frac{2(n-2)}{(p-1)^{2}}\left(p-\frac{n}{n-2}\right)\right)^{1 /(p-1)},
$$

$\left(\mathrm{E}_{2}\right)$ When $p>(n+2) /(n-2)$, all radial positive regular solutions of $(0.4)$ are included in a family $\left\{u_{\alpha}\right\}_{\alpha>0}$ with $u_{\alpha}(r)$ decreasing in $r, u_{\alpha}(0)=\alpha$, $r^{2 /(p-1)} u_{\alpha}(r) \rightarrow L$ as $r \rightarrow+\infty$,

$\left(\mathrm{E}_{3}\right)$ When $p=(n+2) /(n-2)$, all radial positive regular solutions of $(0.4)$ are included in the family $\left\{u_{\lambda}^{E}\right\}_{\lambda>0}, u_{\lambda}^{E}(r)=\left(\lambda \sqrt{n(n-2)} /\left(\lambda^{2}+r^{2}\right)\right)^{(n-2) / 2}$.

We point out that when $1<p<(n+2) /(n-2),(0.4)$ has no regular radial solution (even nonradial solutions cannot exist; see [5]).

Now, we can begin to describe our main results. Let

$$
p_{c}= \begin{cases}\frac{(n-2)^{2}-4 n+4 \sqrt{n^{2}-(n-2)^{2}}}{(n-2)(n-10)} & \text { when } n>10, \\ +\infty & \text { when } 3 \leq n \leq 10 .\end{cases}
$$

It is straightforward to verify that $p_{c}>(n+2) /(n-2)$.

Theorem 0.1. Suppose $n \geq 3$.

(i) When $p>n /(n-2)$, if $0 \leq \phi \leq \lambda u_{s}$ for $u_{s}$ in $\left(\mathrm{E}_{1}\right)$ and some $0<\lambda<$ 1 , then (0.1) has a global classical solution $u$ satisfying $0 \leq u \leq \lambda u_{s}$ and $\|u(\cdot, t)\|_{L^{\infty}\left(R^{n}\right)} \leq\left(\left(\lambda^{1-p}-1\right)(p-1)\right)^{-1 /(p-1)} t^{-1(p-1)}$.

(ii) When $n /(n-2)<p<p_{c}$, if $0 \leq \phi \leq u_{s}$, then (0.1) has a global classical solution $u$ such that $0 \leq u \leq u_{s}$ and $\|u(\cdot, t)\|_{L^{\infty}\left(R^{n}\right)} \rightarrow 0$ as $t \rightarrow+\infty$. 
Remark 1. When $p \geq p_{c}$, (ii) is not true because all radial regular steady states of (0.1) remain below $u_{s}$ (see Proposition 3.7) and therefore even if the classical solution exists globally, it may not decay. However, when $p \geq p_{c}$, with the same condition in (ii), we can prove that either $u$ is a global classical solution or $u$ is classical before time $T_{\phi}>0$ and after $T_{\phi}, u$ is a distributional solution. In any case, $0 \leq u \leq u_{s}$ on $R^{n} \times[0,+\infty)$ and hence by the regularity theory for parabolic equations, $u$ is at least classical on $R^{n} \times[0,+\infty) \backslash\{0\} \times[0,+\infty)$. The large time behavior of $u$ in this case remains open.

Remark 2. As Lee and Ni [24] observed, when liminf $|x| \rightarrow+\infty|x|^{2 /(p-1)} \phi(x)>0$ and if the classical solution exists globally, then $\|u(\cdot, t)\|_{\infty} \geq c t^{-1 /(p-1)}$ for some $c>0$. Thus in (i) the $L^{\infty}$ decay rate of $u$ is sharp if, in addition to the condition in (i), $\phi(x) \geq c_{1}|x|^{-2 /(p-1)}$ at $x=\infty$.

Remark 3. For $\alpha \geq 0$, let $w_{\alpha}(x)=|x|^{2 /(p-1)-\alpha}$. In both (i) and (ii), since $u \leq u_{s}$, we can see that actually if $\alpha>0$,

$$
\|u(\cdot, t)\|_{C_{w_{\alpha}}\left(R^{n}\right)}=\left\|u(\cdot, t) w_{\alpha}(\cdot)\right\|_{L^{\infty}\left(R^{n}\right)} \rightarrow 0 \text { as } t \rightarrow+\infty .
$$

(Note $u_{s}$ and hence $u$ decay faster than $w_{\alpha}$ in $x$ if $\alpha>0$.) From (i), if $\phi \in B_{L}=\left\{\phi \in C_{w_{0}}\left(R^{n}\right) \mid \phi \geq 0\right.$ and $\left.\|\phi\|_{c_{w_{0}}\left(R^{n}\right)}<L\right\}$, then the global flow $u$ initiated at $\phi$ stays in $B_{L}$ and hence $B_{L}$ is an invariant set. Also by (i), if $\phi \geq 0$

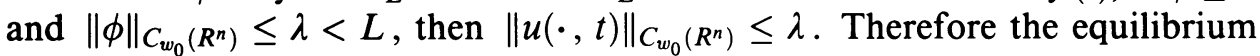
$u_{0} \equiv 0$ is stable with respect to the $C_{w_{0}}\left(R^{n}\right)$ topology (for the definition, see [17]). We point out that if we interpret $u^{p}$ in $(0.1)$ by $|u|^{p-1} u$, in virtue of the comparison principle, the above assertions still hold with obvious modification even for solutions that are not nonnegative.

Remark 4. From (i) and (ii), our answer to (Q) is that when $n \geq 3$ and $n /(n-2)<p<p_{c}, b$ can be as large as $L$ (as given in $\left.\left(E_{1}\right)\right)$ for any $k>0$; when $n \geq 3$ and $p \geq p_{c}, b$ can be arbitrarily close to $L^{-}$.

In this paper, we also obtain some results in the converge direction of Theorem 0.1 .

Theorem 0.2. Suppose $n \geq 3$.

(i) When $p \geq p_{c}$, if $\liminf _{|x| \rightarrow+\infty}|x|^{2 /(p-1)} \phi(x)>L$, then the local classical solution $u$ blows up in finite time.

(ii) When $n /(n-2)<p<p_{c}$, there exists a family $\left\{\phi_{\beta}\right\}_{\beta>0}$, where $\phi_{\beta}$ is radial and decreasing in $r=|x|, \phi_{\beta}(0)=\beta, \phi_{\beta} \equiv u_{s}$ near $x=\infty$, $\phi_{\beta}>u_{s}$ on some ring depending on $\beta$, such that if $\phi \geq \phi_{\beta}$ for some $\beta>0$, then the local classical solution of $(0.1)$ blows up in finite time.

Remark. Combining Remark 4 of Theorem 0.1 and (i) of Theorem 0.2, we see that the least upper bound of $b$ in $(\mathrm{Q})$ is $L$ when $n \geq 11$ and $p \geq p_{c}$. The least upper bound of $b$ for other cases remains unknown. However, (ii) of both Theorem 0.1 and Theorem 0.2 indicate that when $n \geq 3$ and $n /(n-2)<p<$ $p_{c}$, near $u_{s}$ we are in a delicate situation.

At this point, combining Theorem 0.1 and Theorem 0.2 with the previous results in the literature, we can look at $(0.1)$ from the following point of view. Let $p$ be fixed, say $p=2$. Then the results of Fujita, Hayakawa, Kobayashi et al. say that when $n=1,2(p \leq(n+2) / n)$, the local solution of $(0.1)$ blows up in finite time no matter how close $\phi$ is to the equilibrium $u_{0} \equiv 0$, hence $u_{0}$ 
is not stable. When $n \geq 3(2=p>(n+2) / n)$, by results of Lee and $\mathrm{Ni}, u_{0}$ attracts, with rate $c t^{-1}$, the "heat" flow in $C_{B}\left(R^{n}\right)$ initiated at $\phi$ if $\phi$ is close to $u_{0}$ in some weighted $L^{\infty}\left(R^{n}\right)$ topology (more precisely, if

$$
\phi \in B_{b, k}=\left\{\phi \in C\left(R^{n}\right) \mid \phi \geq 0,\left\|\phi(\cdot)\left(k+|\cdot|^{2}\right)\right\|_{L^{\infty}\left(R^{n}\right)} \leq b\right\}
$$

with $k>0, b>0$ and small). Thus when $n \geq 3, u_{0} \equiv 0$ is "stable" in some sense. Now by Remark 3 of Theorem 0.1 , when $n \geq 5(2=p>n /(n-2)) u_{0}$ is stable with respect to the $C_{w_{0}}\left(R^{n}\right)$ topology (recall that $C_{w_{\alpha}}\left(R^{n}\right)=\{\phi \in$ $\left.C\left(R^{n}\right) \mid\left\|\phi(\cdot) w_{\alpha}(\cdot)\right\|_{L^{\infty}\left(R^{n}\right)}<+\infty\right\}$, where $w_{\alpha}(x)=|x|^{2-\alpha}$ when $\left.p=2\right), B_{L}=$ $\left\{\phi \in C_{w_{0}}\left(R^{n}\right) \mid \phi \geq 0,\|\phi\|_{C_{w_{0}}\left(R^{n}\right)}<L\right\}$ is an invariant set of the "heat" flow and $u_{0} \equiv 0$ attracts, in the weaker $C_{w_{\alpha}}\left(R^{n}\right)(\alpha>0)$ topology, all flows initiated in $B_{L}$. From (i) of Theorem 0.2 , when $n \geq 17\left(2=p \geq p_{c}\right)$, the flow may even fail to be global if $\phi$ is outside $B_{L}$ (this is also true if $\phi$ is outside $B_{\lambda L}$ for $\lambda$ large when $5 \leq n \leq 16$; see $(0.3)$ ). Thus when $n \geq 17$ we can say, in some sense, that the "radius of the invariant set" is $L$. Note that when $n \uparrow+\infty$,

$$
L=\left.\left(\frac{2(n-2)}{(p-1)^{2}}\left(p-\frac{n}{n-2}\right)\right)^{1 /(p-1)}\right|_{p=2}=2 n-8 \uparrow+\infty
$$

Observe also that the decay rate $\left(\lambda^{-1}-1\right) t^{-1}$ of $L^{\infty}\left(R^{n}\right)$ norm of the global solution initiated at $\phi \in B_{\lambda L}(0<\lambda<1)$ (see (i) of Theorem 0.1 ) is independent of $n$. All these points seem to indicate that when $n$ is large and increasing, the "stability" of $u_{0} \equiv 0$ is also "increasing". This can be interpreted intuitively that when $n$ is large, there are more dimensions in which "heat" can dissipate away and hence for large initial value, "heat" can diffuse to $\infty$ in $R^{n}$ so much that the "temperature" eventually drops down to zero, while when $n$ is smaller, "heat" cannot dissipate away fast enough and blow-up occurs, and in fact when $n=1,2$, blow-up always happens no matter how small the initial value is.

The following result is of independent interest. It implies that any nontrivial radial regular equilibrium of $(0.1)$ is unstable w.r.t. the $C_{w_{0}}\left(R^{n}\right)$ topology when $p>(n+2) /(n-2)$ and w.r.t. a finer topology when $p=(n+2) /(n-2)$.

Theorem 0.3. Suppose $n \geq 3$ and $p \geq(n+2) /(n-2)$.

(i) If $0 \leq \phi \leq \lambda u_{\alpha}$ for some $0<\lambda<1$ and some radial equilibrium $u_{\alpha}$ (defined in $\left(\mathrm{E}_{2}\right)$ and $\left(\mathrm{E}_{3}\right)$ ), then $(0.1)$ has a global classical solution $u$ satisfying

$$
\|u(\cdot, t)\|_{L^{\infty}\left(R^{n}\right)} \leq\left(\left(\lambda^{1-p}-1\right)(p-1)\right)^{-1 /(p-1)} t^{-1 /(p-1)} .
$$

(ii) If $\phi \geq \lambda u_{\alpha}$ for some $\lambda>1$ and some $u_{\alpha}$ (in $\left(\mathrm{E}_{2}\right)$ and $\left(\mathrm{E}_{3}\right)$ ), then the local classical solution blows up in finite time.

Remark 1. It is interesting to observe and easy to prove by using the continuity of the solution with respect to the initial value that if $\phi=\lambda u_{\alpha}$ with $\lambda>1$, then the "life span" $T_{\lambda u_{\alpha}}$ of local classical solution $u$ of $(0.1)$ satisfies $T_{\lambda u_{\kappa}} \uparrow+\infty$ as $\lambda \downarrow 1$.

Remark 2. When $\lambda$ is small, the decay rate of $u$ in (i) was obtained in [24].

We remark the Theorems $0.1-0.3$ follow from our general results, which assert that if the initial value $\phi$ in $(0.1)$ is $\lambda \psi$ where $\psi \geq 0$ is a radial continuous distributional upper (lower) solution of (0.4) and $0<\lambda<(>) 1$, then 
for $p>n /(n-2)$ the solution of $(0.1)$ decays as stated in (i) of Theorem 0.1 (or blows up in finite time); moreover, if $\lambda=1$ and $\psi$ is not an equilibrium, then for $(n+2) /(n-2) \leq p<p_{c}$, the solution of $(0.1)$ decays (or blows up in finite time).

We should mention a recent work of Bandle and Levine which is related to Theorem 0.1 . In [3], they consider the first initial-boundary value problem

$$
\begin{aligned}
& u_{t}=\Delta u+u^{p}, \quad p>1, \\
& \left.u(\cdot, t)\right|_{\partial D}=0, \\
& \left.u\right|_{t=0}=\phi \geq 0, \quad \phi \in C_{0}(\bar{D})=\left\{\phi \in C(\bar{D})|\phi|_{\partial D}=0\right\},
\end{aligned}
$$

where $D=\Omega \times(0, \infty) \subset R^{n}, \Omega \subset S^{n-1}$ is a open connected manifold with boundary. After obtaining an interesting Fujita-type nonexistence result, they prove that if

$$
p_{1}(\Omega)<p<\left\{\begin{array}{ll}
\frac{n+1}{n-3}, & n>3 \\
\infty, & n=2,3
\end{array} \quad\left(1<p_{1}(\Omega)<\frac{n}{n-2}\right),\right.
$$

there exists a singular steady state $u_{s}^{D}$ and if $\left.u\right|_{t=0}=\phi \leq u_{s}^{D}$, the classical solution $u$ is global and, when $p_{1}(\Omega)<p<(n+2) /(n-2), u$ decays to zero pointwise as $t \rightarrow+\infty$. They also considered the case when $D$ is exterior to a bounded domain and $0 \in D^{C}$, and proved that if $n \geq 3, p>n /(n-2)$ and $\left.u\right|_{t=0} \leq u_{s}$ (as in $\left.\left(\mathrm{E}_{1}\right)\right)$, then $u$ is a global classical solution, moreover, if $n /(n-2)<p \leq(n+2) /(n-2), u$ decays to zero pointwise as $t \rightarrow+\infty$.

By a simple comparison argument, using Theorem 0.1 and Remark 1 following, we make concerning $(0.5)$ the following observations. When $D$ is either a cone or an exterior domain as mentioned above (but we allow $0 \in \bar{D}$ ), then Theorem 0.1 is true for (0.5); moreover, when $n \geq 3, p \geq p_{c}, 0 \notin \bar{D}$, and $0 \leq \phi \leq u_{s},(0.5)$ still has a global classical solution.

Remark 1. This result does not cover the case when $p_{1}(\Omega)<p \leq n /(n-2)$ and $n \geq 3$, and the case when $n=2$. These cases are covered by Bandle and Levine [3] when $D$ is a cone. However, when $n \geq 3$, our result covers the cases that remained open in [3] and provides the decay rates for global solutions.

Remark 2. By (3.3) and (3.6) in [3], $u_{s}^{D}(r, \theta)=r^{-2 /(p-1)} \alpha(\theta)$ where $\Delta_{\theta} \alpha-$ $L^{p-1} \alpha+\alpha^{p}=0$ in $\Omega, \alpha=0$ on $\partial \Omega$. Suppose $\alpha\left(\theta_{0}\right)=\max _{\theta \in \bar{\Omega}} \alpha$, then $\Delta_{\theta} \alpha\left(\theta_{0}\right) \leq 0$; hence $-L^{p-1} \alpha\left(\theta_{0}\right)+\alpha^{p}\left(\theta_{0}\right) \geq 0, \alpha\left(\theta_{0}\right) \geq L$ when $p>$ $n /(n-2)$. Now by the strong maximum principle (the manifold version; see [2]), $\alpha\left(\theta_{0}\right)>L$. Thus the graph of $u_{s}^{D}$ intersects (transversely) that of $u_{s}$ when $p>n /(n-2)$. So in case $D$ is a cone, the result in [3] mentioned above and our result, when they overlap, are not comparable.

Remark 3. Theorem 0.1 can be applied to $(0.5)$ when $D$ is of other geometric types.

In this paper, we also consider the following more general version of $(0.1)$ :

$$
\begin{array}{ll}
u_{t}=\Delta u+|x|^{l} u^{p}, & x \in R^{n}, t>0, \text { and } u \geq 0, \\
\left.u\right|_{t=0}=\phi \in C\left(R^{n}\right), & \phi \geq 0 \text { and } \phi \not \equiv 0,
\end{array}
$$

where $p>1, l>-2$, and $N \geq 3$. When $l<0$ it is generally impossible to obtain a classical solution for $(0.6)$. Therefore we consider only those " $C_{B}$-mild" 
solutions of (0.6), i.e., flows in $C_{B}\left(R^{n}\right)$, and by contraction mapping method, we prove the local existence of $C_{B}$-mild solution for (0.6) with $-2<l<0$ and $\phi \in C_{B}\left(R^{n}\right)$. When $l \geq 0$, we prove that if $0 \leq \phi(x) \leq C|x|^{-l /(p-1)}$ on $R^{n}$, then (0.6) has a local classical solution with the same decay rate in the $x$ variable, while when $\phi$ decays more slowly than $|x|^{-l /(p-1)},(0.6)$ does not possess any local solution which can be dominated by any polynomial of $|x|$ (see Theorem 2.5).

As for global existence, we observe that by modifying the arguments of Lee and $\mathrm{Ni}$ [24], one can prove that if $(0.6)$ has a global solution, then necessarily $\lim \inf _{|x| \rightarrow+\infty}|x|^{(2+l) /(p-1)} \phi(x)<+\infty$. Also, by a Fujita type blow-up result of Bandle and Levine [4] for the exterior domain case and a comparison argument, when $1<p<(n+2+l) / n$, the local solution of (0.6) blows up in finite time if $\phi$ is nontrivial. When $p>(n+2+l) / n$ and $l \geq 0$, by the proof of Theorem 3.1 in [4], if $\phi$ is dominated by a small multiple of a Gaussian, (0.6) possesses a global solution $u$ whose $L^{\infty}\left(R^{n}\right)$ norm decays like $t^{-\gamma}$ where $\gamma=(2+l) / 2(p-1)$ (it seems that the proof there cannot be applied to $(0.6)$ when $-2<l<0)$. In this paper, for $p>(n+l) /(n-2)(>(n+2+l) / n)$, for larger initial values and even for $-2<l<0$, we establish a global existence and large-time behavior result similar to Theorem 0.1 . Results similar to Theorem 0.2 and Theorem 0.3 are also obtained for $(0.6)$. Before we describe them more precisely, again we need to recall some brief background on the equilibria of (0.6). Henceforth, we redefine that

$$
\begin{aligned}
L & =\left(\frac{(2+l)(n-2)}{(p-1)^{2}}\left(p-\frac{n+l}{n-2}\right)\right)^{1 /(p-1)}, \\
p_{c} & =\frac{(n-2)^{2}-2(l+2)(n+l)+2(l+2) \sqrt{(n+l)^{2}-(n-2)^{2}}}{(n-2)(n-10-4 l)}, \quad n>10+4 l, \\
& =+\infty, \quad n \geq 3 \text { and } n \leq 10+4 l,
\end{aligned}
$$

and:

$\left(\mathrm{E}_{1}\right)_{l}$ When $p>(n+l) /(n-2), u_{s}(r)=L r^{-(2+l) /(p-1)}(r=|x|)$ is a singular stationary solution of $(0.6)$.

$\left(\mathrm{E}_{2}\right)_{l}$ When $p>(n+2+2 l) /(n-2)$, all radial positive regular stationary solutions of (0.6) are included in a family $\left\{u_{\alpha}\right\}_{\alpha>0}$ with $u_{\alpha}(r)$ decreasing in $r, u_{\alpha}(0)=\alpha, r^{(2+l) /(p-1)} u_{\alpha}(r) \rightarrow+\infty$.

$\left(\mathrm{E}_{3}\right)_{l}$ When $p=(n+2+2 l) /(n-2)$, all radial positive stationary solutions of (0.6) are included in a family $\left\{u_{\lambda}^{E}\right\}_{\lambda>0}$,

$$
u_{\lambda}^{E}(r)=\left(\frac{\lambda \sqrt{(n+l)(n-2)}}{\lambda^{2}+r^{2+l}}\right)^{(n-2) /(2+l)} .
$$

Our main results for global existence and nonexistence of solutions (0.6) are as follows.

Theorem 0.1'. Suppose $n \geq 3$.

(i) When $(n+l) /(n-2)<p<p_{c}$, if $0 \leq \phi \leq u_{s}$ (defined in $\left.\left(\mathrm{E}_{1}\right)_{l}\right)$, then (0.6) has a global solution $u$ such that $0 \leq u \leq u_{s}$ and $\|u(\cdot, t)\|_{L^{\infty}\left(R^{n}\right)}$ 
$\rightarrow 0$ as $t \rightarrow+\infty$; the same is true with $0 \leq u \leq \lambda u_{s}$ when $p>$ $(n+l) /(n-2)$ and $0 \leq \phi \leq \lambda u_{\text {s }}$ for some $0<\lambda<1$.

(ii) When $p \geq \max \{(n+4+2 l) / n,(n+2+2 l) /(n-2)\}$, there exists a small $\lambda>0$ such that if $0 \leq \phi(x) \leq \lambda(1+|x|)^{-(2+l) /(p-1)}$ on $R^{n}$, then $(0.6)$ has a global solution $u$ with $\|u(\cdot, t)\|_{L^{\infty}\left(R^{n}\right)} \leq M t^{-(2+l) / 2(p-1)}$.

Remark. We are unable to obtain the decay rate for global solutions when $\lambda$ is close to 1 and $p$ is small, as we can do when $l=0$ in (0.6). However, the observations in Remark 3 of Theorem 0.1 about the stability of $u_{0} \equiv 0$ still hold with obvious modifications.

Theorem 0.2' . Suppose in (0.6) that $\phi$ is bounded and when $l \geq 0,0 \leq \phi(x) \leq$ $c|x|^{-l /(p-1)}$ on $R^{n}$. Then (0.6) has a local solution which blows up in finite time if

(i) when $p \geq p_{c}, \liminf _{|x| \rightarrow+\infty}|x|^{(2+l) /(p-1)} \phi(x)>L$, or

(ii) when $(n+l) /(n-2)<p<p_{c}, \phi \geq \phi_{\beta}$ for some $\phi_{\beta}$ described in (ii) of Theorem 0.2 but with $u_{s}$ replaced by the $u_{s}$ in $\left(\mathrm{E}_{1}\right)_{l}$.

Theorem 0.3'. Suppose that $p \geq(n+2+2 l) /(n-2)$.

(i) If $0 \leq \phi \leq \lambda u_{\alpha}$ for some $0<\lambda<1$ and some radial equilibrium $u_{\alpha}$ in $\left(\mathrm{E}_{2}\right)_{l}$ and $\left(\mathrm{E}_{3}\right)_{l}$, then $(0.6)$ has a global solution $u$ satisfying $0 \leq u \leq \lambda u_{\alpha}$ and $\|u(\cdot, t)\|_{L^{\infty}\left(R^{n}\right)} \rightarrow 0$.

(ii) If $\phi \geq \lambda u_{\alpha}$ for some $\lambda>1$ and some $u_{\alpha}$ in (i), $\phi$ is bounded and, when $l \geq 0,0 \leq \phi(x) \leq C|x|^{-l /(p-1)}$ on $R^{n}$, then (0.6) has a local solution which blows up in finite time.

We wish to point out that in Theorem $0.2^{\prime}$ and (ii) of Theorem $0.3^{\prime}$, by the results in $\S 2$, the $L^{\infty}$ norm of the local solution $u$ blows up in finite time when $-2<l<0$ and $\left\|u(\cdot, t)(1+|\cdot|)^{l /(p-1)}\right\|_{L^{\infty}\left(R^{n}\right)}$ blows up in finite time when $l \geq 0$.

Finally, we remark that Theorem $0.1^{\prime}$ can also be used to obtain global existence for various related first boundary-initial value problems.

The organization of this paper is as follows. Section 1 contains some preliminary lemmas. In $\S 2$, we establish local existence for $(0.6)$ and give some properties of local solutions. In $\S 3$, existence and nonexistence of global solutions for $(0.6)$ are studied. In $\S 4$, we derive the decay rate of global solutions in various cases. Section 5 includes some observations about first boundaryinitial value problems (including (0.5)) related to $(0.6)$. Theorem 0.1 follows from Theorem 3.9 and Corollary 4.2, while Theorem $0.1^{\prime}$ is a consequence of Theorem 3.9 and Theorem 4.9. Theorem 0.2 and Theorem $0.2^{\prime}$ are implied by Theorem 3.11. Finally, Theorem 0.3 is a consequence of Theorem 4.1 and (iv) of Theorem 3.11, and Theorem $0.3^{\prime}$ follows from (iv) of Theorem 3.9 and (iv) of Theorem 3.11.

\section{Preliminaries}

Suppose $D$ is an unbounded domain in $R^{n}$ with $\partial D$ satisfying the exterior sphere condition. Let $T>0, \Omega=D \times(0, T)$, and $\Gamma=\partial D \times(0, T) \cup \bar{D} \times\{0\}$. For a given function $\psi \in C(\Gamma)$, we consider the following boundary value 
problem:

$$
\begin{aligned}
& u_{t}=\Delta u+f(x, t, u) \text { in } \Omega, \\
& \left.u\right|_{\Gamma}=\psi .
\end{aligned}
$$

Definition 1.1. We call a function $u$ a continuous weak (c.w.) upper (lower) solution of (1.1) if $u$ is continuous on $\bar{\Omega},\left.u\right|_{\Gamma} \geq(\leq) \psi$ and $u_{t} \geq(\leq) \Delta u+$ $f(x, t, u)$ in the distributional sense, i.e., for any $\eta \in C^{2,1}(D \times[0, T])$ with $\eta \geq 0$ and $\operatorname{supp} \eta(\cdot, t) \Subset D$ for all $t \in[0, T]$,

$$
\begin{aligned}
& \left.\int_{D} u(x, t) \eta(x, t) d x\right|_{t=0} ^{t=T_{1}} \\
& \quad \geq(\leq) \int_{0}^{T_{1}} \int_{D}\left[u(x, s)\left(\Delta \eta+\eta_{t}\right)(x, s)+\eta(x, s) f(x, s, u)\right] d x d s,
\end{aligned}
$$

if $T_{1} \in[0, T]$. If $u$ is a c.w. upper and also a c.w. lower solution of (1.1), we say $u$ is a continuous weak (c.w.) solution. We call a function $u$ a classical solution of $(1.1)$ if $u \in C^{2,1}(\Omega) \cap C(\bar{\Omega})$ and (1.1) is satisfied.

The monotonicity method for (1.1) when $D$ is bounded was settled by Sattinger [33] (more general operators were considered). When $D$ is unbounded, the method is also well known. However, it seems difficult to find a proof in the literature. Therefore, for the sake of completeness, we include such a proof here. We shall content ourselves with just dealing with (1.1), though the result can be generalized to more general parabolic operators (second order) and boundary operators.

Lemma 1.2. Suppose that $f(x, t, u)$ is continuous on $\bar{\Omega} \times R$, locally Hölder continuous in $(x, t) \in \Omega$ locally uniformly with respect to $u$, locally Lipschitz continuous in $u$ uniformly for $(x, t)$ in any bounded subset of $\Omega$. Assume that $\bar{u}$ and $\underline{u}$ are continuous weak upper and lower solutions of (1.1) with $\bar{u} \geq \underline{u}$ on $\bar{\Omega}$. Then (1.1) has a classical solution $u$ satisfying $\underline{u} \leq u \leq \bar{u}$ on $\bar{\Omega}$.

Proof. First, when $D$ is bounded, the conclusion is true. This is basically covered by [33]. But since our conditions are slightly different from Sattinger's, we present a proof here using the ideas in [33] as follows. Without loss of generality, suppose $f$ is nondecreasing with respect to $u \in\left[\min _{\Omega} \underline{u}, \max _{\Omega} \bar{u}\right]$ (otherwise, for a large $k$, replace $f$ by $k u+f(x, t, u)$ and $\Delta$ by $\Delta-k$ in the following argument).

Let $G(x, y, t)$ be the Green's function of

$$
\begin{aligned}
& u_{t}=\Delta u, \quad(x, t) \in \Omega, \\
& \left.u\right|_{\Gamma}=0 .
\end{aligned}
$$

Let $u_{\psi}$ be the classical solution of

$$
u_{t}=\Delta u,\left.\quad u\right|_{\Gamma}=\psi,
$$

$G$ and $u_{\psi}$ can be obtained by the Perron method because of the regularity of $\partial D$ (see [9]). Define $u_{0} \equiv \underline{u}, u_{1} \equiv u_{\psi}+T\left(u_{0}\right)$ where

$$
T\left(u_{0}\right)(x, t)=\int_{0}^{t} \int_{D} G(x, y, t-s) f(y, s, \underline{u}(y, s)) d y d s .
$$


We claim that $T\left(u_{0}\right)$ is a continuous weak solution of

$$
\begin{aligned}
& u_{t}=\Delta u+f(x, t, \underline{u}) \text { on } \Omega, \\
& \left.u\right|_{\Gamma}=0 .
\end{aligned}
$$

To see this, take a sequence $g_{k} \in C^{1}(\bar{\Omega})$ such that $g_{k} \rightarrow h$ in $C(\bar{\Omega})$ where $h(x, t)=f(x, t, \underline{u}(x, t))$. By classical theory

$$
v_{k}(x, t)=\int_{0}^{t} \int_{D} G(x, y, t-s) g_{k}(y, s) d y d s
$$

is the classical solution of

$$
\begin{aligned}
& v_{t}=\Delta v+g_{k}(x, t) \quad \text { on } \Omega, \\
& \left.v\right|_{\Gamma}=0 .
\end{aligned}
$$

Since $g_{k}$ is uniformly bounded on $\Omega$, i.e., $\left|g_{k}\right| \leq C_{1}<+\infty$ on $\bar{\Omega}$, then by the comparison principle, $\left|v_{k}\right| \leq w_{1}$ on $\bar{\Omega}$ where $w_{1}$ is the solution of (1.2) with $f(x, t, \underline{u})$ replaced by $C_{1}$, in particular, $v_{k}$ is uniformly bounded on $\bar{\Omega}$. Now by the $L^{p}$ interior estimate for parabolic equations, for any $q>1$ and any $\Omega^{\prime} \Subset$ $\Omega,\left\|v_{k}\right\|_{w_{q}^{2,1}\left(\Omega^{\prime}\right)} \leq M\left(\Omega^{\prime}\right)<+\infty$ where $M\left(\Omega^{\prime}\right)$ is independent of $k$. By the embedding theorems and a diagonalization argument, there exists a subsequence of $v_{k}$ (still denote it by $\left\{v_{k}\right\}$ ) such that $v_{k} \rightarrow$ some $v$ in $C_{\mathrm{loc}}^{2-\alpha, 1-\alpha / 2}(\Omega)$ for a $0<\alpha<1$. Since $\left|v_{k}\right| \leq w_{1}$ and $\left.w_{1}\right|_{\Gamma}=0$, then $v \in C(\bar{\Omega})$ and $\left.v\right|_{\Gamma}=0$. Also, using Definition 1.1, it is easy to check that $v$ is a distributional solution of (1.2). On the other hand, by the Lebesgue Dominated Convergence Theorem, $v_{k} \rightarrow T\left(u_{0}\right)$ pointwise in $\Omega$. Thus $T\left(u_{0}\right)=v$ and $T\left(u_{0}\right)$ is a c.w. solution of (1.2).

Now, $u_{1}=u_{\psi}+T\left(u_{0}\right)$ is a c.w. solution of

$$
\begin{aligned}
& u_{t}=\Delta u+f(x, t, \underline{u}) \text { on } \Omega, \\
& \left.u\right|_{\Gamma}=\psi .
\end{aligned}
$$

Observe $\underline{u}(\bar{u})$ is a c.w. lower (upper) solution of (1.3) (note $f(x, t, \underline{u}) \leq$ $f(x, t, \bar{u}))$. By the strong maximum principle for weakly subparabolic functions (see [8]), we have $u_{1} \geq \underline{u}$ and $u_{1} \leq \bar{u}$ on $\bar{\Omega}$. Define $u_{k}=u_{\psi}+T\left(u_{k-1}\right)$ then similarly as above, we have $\underline{u} \leq u_{1} \leq u_{2} \leq \cdots \leq u_{k} \leq \bar{u}$ on $\bar{\Omega}$. Let $u=\lim _{k \rightarrow+\infty} u_{k}$, then by the Lebesgue Dominated Convergence Theorem, $\lim _{k \rightarrow+\infty} T\left(u_{k}\right)=T(u)$ and by the same reasoning regarding $T\left(u_{0}\right)$ in the previous paragraph, we can prove that $u=u_{\psi}+T(u)$ is a c.w. solution of (1.1). A bootstrap argument then implies that $u$ is a classical solution of (1.1). Obviously $\underline{u} \leq u \leq \bar{u}$. We have completed the proof of Lemma 1.2 in case of bounded $D$.

Now suppose $D$ is unbounded. In $R^{n}$, take an increasing sequence of bounded domains $\left\{D_{k}\right\}$ such that each $\partial D_{k}$ satisfies the exterior sphere condition, $\bigcup D_{k}=D$ and $\bigcup\left(\partial D_{i} \cap \partial D\right)=\partial D$. Let $\Omega_{k}=D_{k} \times(0, T)$ and $\Gamma_{k}$ be the parabolic boundary of $\Omega_{k}$. Let $\tilde{\psi}$ be a continuous extension of $\psi$ in $R^{n+1}$. On $\bar{\Omega}$ define $\Phi \equiv \max (\min (\bar{u}, \tilde{\psi}), \underline{u})$. Clearly $\left.\Phi\right|_{\Gamma}=\left.\psi\right|_{\Gamma}, \underline{u} \leq \Phi \leq \bar{u}$ on $\Omega$. Consider

$$
\begin{aligned}
& u_{t}=\Delta u+f(x, t, u), \quad(x, t) \in \Omega_{k}, \\
& \left.u\right|_{\Gamma_{k}}=\left.\Phi\right|_{\Gamma_{k}} .
\end{aligned}
$$


By the conclusion for bounded domains proved above, (1.4) $k_{k}$ has a classical solution $u_{k}$ with $\underline{u} \leq u_{k} \leq \bar{u}$ for each $k \geq 1$. Applying $L^{p}$ interior estimates and then Schauder interior estimates to $u_{k}$, we have for any $\Omega^{\prime} \Subset \Omega$,

$$
\left\|u_{k}\right\|_{C^{2+\alpha, 1+\alpha / 2}\left(\Omega^{\prime}\right)} \leq M\left(\Omega^{\prime}\right),
$$

where $M\left(\Omega^{\prime}\right)$ is independent of $k$ and $0<\alpha<1$. From this and a diagonalization argument, there is a subsequence of $\left\{u_{k}\right\}$ (still denote it by $\left\{u_{k}\right\}$ ) such that $u_{k} \rightarrow u$ in $C_{\mathrm{loc}}^{2,1}(\Omega)$. Obviously $u$ satisfies the differential equation in (1.1) and $\underline{u} \leq u \leq \bar{u}$ on $\Omega$.

It remains to prove $u \in C(\bar{\Omega})$ and $\left.u\right|_{\Gamma}=\psi\left(=\left.\Phi\right|_{\Gamma}\right)$. We use the standard barrier method. Take and fix any $Q \in \Gamma$. Then there exists $k_{0}>0$ such that $Q \in \Gamma_{k}$ for any $k \geq k_{1}$. Find a barrier $w_{Q}$ (the existence is justified by the regularity of $\left.\partial D_{k_{1}}\right)$ such that $w_{Q} \in C\left(\bar{\Omega}_{k_{1}}\right) \cap C_{\mathrm{loc}}^{2,1}\left(\Omega_{k_{1}}\right), w_{Q}(P)>0$ for $P \in \bar{\Omega}_{k_{1}}$ and $P \neq Q, w_{Q}(Q)=0$ and $\partial w_{Q} / \partial t-\Delta w_{Q} \geq 1$ on $\Omega_{k_{1}}$ (see [9]). Let $v_{\varepsilon}^{+}=\psi(Q)+\varepsilon+m w_{Q}, v_{\varepsilon}^{-}=\psi(Q)-\varepsilon-m w_{Q}$, where $\varepsilon>0$ is fixed, $m$ a constant to be chosen. Since $u_{k}$ and $f\left(x, t, u_{k}\right)\left(k \geq k_{1}\right)$ are uniformly bounded on $\bar{\Omega}_{k_{1}}$ and $\left.u_{k}\right|_{\Gamma_{k_{1}}}=\left.\Phi\right|_{\Gamma_{k_{1}}}$ near $Q$ for large $k$, there exists an $m_{\varepsilon}$ such that on $\Omega_{k_{1}}$ for each $k \geq k_{1}$,

$$
v_{\varepsilon}^{+}\left|\Gamma_{k_{1}} \geq u_{k}\right|_{\Gamma_{k_{1}}} \geq\left. v_{\varepsilon}^{-}\right|_{\Gamma_{k_{1}}}
$$

and

$$
\left(\frac{\partial}{\partial t}-\Delta\right) v_{\varepsilon}^{+} \geq\left(\frac{\partial}{\partial t}-\Delta\right) u_{k} \geq\left(\frac{\partial}{\partial t}-\Delta\right) v_{\varepsilon}^{-}
$$

By the maximum principle, $v_{\varepsilon}^{+} \geq u_{k} \geq v_{\varepsilon}^{-}$on $\bar{\Omega}_{k_{1}}$. Thus

$$
\left|u_{k}(P)-\psi(Q)\right| \leq \varepsilon+m_{\varepsilon} w_{Q}(P)
$$

for $P \in \Omega_{k_{1}}$ and $k \geq k_{1}$. Letting $k \rightarrow+\infty$, we have

$$
|u(P)-\psi(Q)| \leq \varepsilon+m_{\varepsilon} w_{Q}(P) \text { for } P \in \Omega_{k_{1}} .
$$

Hence

$$
\limsup _{\Omega_{k_{1}} \ni P \rightarrow Q}|u(P)-\psi(Q)| \leq \varepsilon .
$$

Therefore $u \in C(\bar{\Omega})$ and $\left.u\right|_{\Gamma}=\psi$.

Next, we recall a comparison principle of Phragmèn-Lindelöf type for (1.1).

Lemma 1.3. Suppose $\bar{u}$ and $\underline{u}$ are continuous weak upper and lower solutions of $(1.1)$ and $(\bar{u}-\underline{u})(x, t) \geq-B \exp \left[\beta|x|^{2}\right]$ on $\Omega$ with $B$ and $\beta>0$. Assume $f(x, t, \bar{u}(x, t))-f(x, t, \underline{u}(x, t)) \geq C(x, t)(\bar{u}-\underline{u})(x, t)$ where $C \in C_{\text {loc }}^{\alpha, \alpha / 2}(\Omega)$ and $C(x, t) \leq C_{0}\left(|x|^{2}+1\right)$ on $\Omega$ for some $C_{0}>0$. Then $\bar{u} \geq \underline{u}$ on $\bar{\Omega}$.

Remark. This lemma can be proved essentially by the same argument involved in the proof of Theorem 9 in Chapter 2 in [9] (see also Theorem 10 in Chapter 3 in [32]) except that whenever the classical minimum principle (in bounded domain) is used in [9], we have to use the minimum principle for weak superparabolic functions in [8].

Now we make an observation which simply says that the convolution of two radial and radially nonincreasing functions is still radial and radially nonincreasing. 
Lemma 1.4. Suppose that both $f$ and $g$ are measurable and radial functions in $R^{n}$, and that for each $x \in R^{n}, h(x)=\int_{R^{n}} f(x-y) g(y) d y$ exists as a Lebesgue integral. Then $h$ is also radial. Moreover, if both $f$ and $g$ are radially nonincreasing, so is $h$.

Proof. The first part follows from the fact that for any rotation $A$ in $R^{n}$, $h(x)=h(A x)$, which can be proved by change of variables. To prove the second part, it suffices to prove that for any $\lambda>0, u(x) \geq u\left(x_{\lambda}\right)$ where $x \in$ $\Sigma_{\lambda}=\left\{x \in R^{n} \mid x_{1}<\lambda\right\}, x_{\lambda}=\left(2 \lambda-x_{1}, x^{\prime}\right)$, i.e., $x_{\lambda}$ is the reflection of $x$ about $\partial \Sigma_{\lambda}$. Observe

$$
\begin{aligned}
u(x) & =\left(\int_{\Sigma_{\lambda}}+\int_{\Sigma_{\lambda}^{c}}\right) f(x-y) g(y) d y \\
& =\int_{\Sigma_{\lambda}} f(x-y) g(y) d y+\int_{\Sigma_{\lambda}} f\left(x-y_{\lambda}\right) g\left(y_{\lambda}\right) d y \\
& =\int_{\Sigma_{\lambda}}\left(f(x-y) g(y)+f\left(x-y_{\lambda}\right) g\left(y_{\lambda}\right)\right) d y .
\end{aligned}
$$

Similarly,

$$
u\left(x_{\lambda}\right)=\int_{\Sigma_{\lambda}}\left(f\left(x_{\lambda}-y\right) g(y)+f(x-y) g\left(y_{\lambda}\right)\right) d y \quad\left(\left|x_{\lambda}-y_{\lambda}\right|=|x-y|\right) .
$$

So we just need to show

$$
f(x-y) g(y)+f\left(x-y_{\lambda}\right) g\left(y_{\lambda}\right) \geq f\left(x_{\lambda}-y\right) g(y)+f(x-y) g\left(y_{\lambda}\right)
$$

for $x, y \in \Sigma_{\lambda}$, i.e.,

$$
\left(f(x-y)-f\left(x-y_{\lambda}\right)\right)\left(g(y)-g\left(y_{\lambda}\right)\right) \geq 0 .
$$

This is proved by $\left|x-y_{\lambda}\right| \geq|x-y|\left(x, y \in \Sigma_{\lambda}\right)$ and the properties of $f$ and $g$.

The next lemma concerns the relation between $(0.6)$ and the corresponding "variation of constants formula".

Lemma 1.5. Suppose that $u$ is a continuous weak upper (lower) solution of

$$
\left\{\begin{array}{l}
u_{t}=\Delta u+|x|^{l} u^{p}, \quad x \in R^{n}, t>0, u^{p}=|u|^{p-1} u, \\
\left.u\right|_{t=0}=\phi \in C\left(R^{n}\right),
\end{array}\right.
$$

where $l>-2, p>1$, and $n \geq 3$. Assume that there exist $k, \beta>0$, and $0<\alpha<2$ such that $|u(x, t)| \leq k \exp \left(\beta|x|^{\alpha}\right)$ on $R^{n} \times[0, T]$. Then

$$
u(\cdot, t) \geq(\leq) e^{t \Delta} \phi+\int_{0}^{t} e^{(t-s) \Delta}|\cdot|^{l} u^{p}(\cdot, s) d s
$$

on $R^{n} \times[0, T]$.

Proof. Let

$$
v(x, t)=\left(e^{t \Delta} \phi+\int_{0}^{t} e^{(t-s) \Delta}|\cdot|^{l} u^{p}(\cdot, s) d s\right)(x) .
$$

Claim 1. $v$ is a c.w. solution of

$$
\begin{aligned}
& v_{t}=\Delta v+|x|^{l} u^{p}, \quad x \in R^{n}, t \in(0, T], \\
& \left.v\right|_{t=0}=\phi .
\end{aligned}
$$


By the classical theory (see [9]), $e^{t \Delta} \phi$ is a classical solution of (1.6) without the term $|x|^{l} u^{p}$. Thus to prove Claim 1 , we just need to show that

$$
I(x, t)=\int_{0}^{t} e^{(t-s) \Delta}|\cdot|^{l} u^{p}(\cdot, s) d s
$$

is a c.w. solution of (1.6) with $\phi \equiv 0$. Let

$$
f(x, t)=\left\{\begin{array}{l}
|x|^{l} u^{p}(x, t), \quad(x, t) \in R^{n} \times[0, T], \\
0 \text { otherwise. }
\end{array}\right.
$$

For $a>0$, take $\xi_{a} \in C_{0}^{\infty}\left(R^{n}\right), 0 \leq \xi_{a} \leq 1$, and

$$
\xi_{a}(x)= \begin{cases}0, & |x| \geq a+1 \\ 1, & |x| \leq a\end{cases}
$$

Let $j$ be the standard mollifier on $R^{n+1}$ and $j_{\varepsilon}(x, t)=\left(1 / \varepsilon^{n+1}\right) j(x / \varepsilon, t / \varepsilon)$. Denote $f \xi_{a}$ by $f_{a}, j_{\varepsilon} * f_{a}$ by $f_{a, \varepsilon}$, let

$$
I_{a, \varepsilon}(x, t)=\int_{0}^{t} e^{(t-s) \Delta} f_{a, \varepsilon}(\cdot, s) d s .
$$

Then $f_{a, \varepsilon} \in C_{0}^{\infty}\left(R^{n+1}\right)$ and $f_{a, \varepsilon} \rightarrow f_{a}$ in $L^{q}\left(R^{n} \times[0, T]\right)$ for $1<q<-n / l$ when $-2<l<0$, and $1<q$ when $l \geq 0$, as $\varepsilon \rightarrow 0^{+}$(the condition on $q$ makes $\left.|x|^{l} \in L_{\mathrm{loc}}^{q}\left(R^{n}\right)\right)$. Also, from the classical theory, $I_{a, \varepsilon}$ is the bounded smooth solution of

$$
I_{t}-\Delta I=f_{a, \varepsilon},\left.\quad I\right|_{t=0}=0 .
$$

In virtue of the standard $L^{p}$ theory, $I_{a, \varepsilon} \rightarrow I_{a}$ in $W_{q}^{2,1}\left(R^{n} \times[0, T]\right)$ as $\varepsilon \rightarrow 0^{+}$ where $I_{a}(x, t)=\int_{0}^{t} e^{(t-s) \Delta} f_{a}(\cdot, s) d s$. Thus $I_{a}$ is a $W_{q}^{2,1}\left(R^{n} \times[0, T]\right)$ and hence (by embedding) a $C_{\mathrm{loc}}^{\alpha, \alpha / 2}\left(R^{n} \times[0, T]\right)$ weak solution of (1.7) with $f_{a, \varepsilon}$ replaced by $f_{a}$. From the growth condition on $u$ and by the proof of Claim 2 below, we have $I_{a} \rightarrow I$ locally uniformly on $R^{n} \times[0, T]$ as $a \rightarrow+\infty$. Therefore, $I$ is a c.w. solution of (1.6) with $\phi \equiv 0$.

Claim 2. There exist $\bar{k}$ and $\bar{\beta}>0$ such that $|v(x, t)| \leq \bar{k} \exp \left(\bar{\beta}|x|^{\alpha}\right)$ on $R^{n} \times[0, T]$. We just prove that $I$ satisfies the claim. The fact that $e^{t \Delta} \phi$ also satisfies the claim follows similarly. For any $(x, t) \in R^{n} \times[0, T]$,

$$
\begin{aligned}
|I(x, t)| \leq & \int_{0}^{t} \frac{d s}{(4 \pi(t-s))^{n / 2}} \int_{R^{n}} \exp \left(\frac{-|x-y|^{2}}{4(t-s)}\right)|y|^{l}|u|^{p}(y, s) d y \\
\leq & C \int_{0}^{t} \frac{d s}{(t-s)^{n / 2}} \int_{R^{n}} \exp \left(\frac{-|x-y|^{2}}{4(t-s)}\right)|y|^{l} \exp \left(p \beta|y|^{\alpha}\right) d y \\
\leq & C\left(\int_{0}^{t} \frac{d s}{s^{n / 2}} \int_{|y| \leq 1} \exp \left(\frac{-|x-y|^{2}}{4 s}\right)|y|^{l} d y\right. \\
& \left.\quad+\int_{0}^{t} \frac{d s}{s^{n / 2}} \int_{|y| \geq 1} \exp \left(\frac{-|x-y|^{2}}{4 s}\right) \exp \left(2 p \beta|y|^{\alpha}\right) d y\right) \\
= & C\left(I_{1}+I_{2}\right) .
\end{aligned}
$$


When $l \geq 0, I_{1} \leq t \leq T$, and when $-2<l<0$, by Lemma 1.4,

$$
\begin{aligned}
I_{1} & \leq \int_{0}^{t} \frac{d s}{s^{n / 2}} \int_{|y| \leq 1} \exp \left(\frac{-|y|^{2}}{4 s}\right)|y|^{l} d y \\
& \leq \int_{0}^{t} d s \int_{R^{n}} \exp \left(-\frac{|\zeta|^{2}}{4}\right) s^{l / 2}|\zeta|^{l} d \zeta \\
& \leq C(n, l) T^{1+l / 2} .
\end{aligned}
$$

Thus, in any case, $I_{1} \leq C(n, l)(1+T)^{1+l / 2}$.

$$
\begin{aligned}
I_{2} & =\int_{0}^{t} \frac{d s}{s^{n / 2}} \int_{|y| \geq 1} \exp \left(\frac{-|x-y|^{2}}{4 s}\right) \exp \left(2 p \beta|y|^{\alpha}\right) d y \\
& \leq \int_{0}^{t} d s \int_{R^{n}} \exp \left(\frac{-|\eta|^{2}}{4}\right) \exp \left(2 p \beta|x-\sqrt{s} \eta|^{\alpha}\right) d \eta \\
& \leq \int_{0}^{t} d s \int_{R^{n}} \exp \left(\frac{-|\eta|^{2}}{4}\right) \exp \left(2 p \beta c(\alpha)\left(|x|^{\alpha}+T^{\alpha / 2}|\eta|^{\alpha}\right)\right) d \eta \\
& \leq C(n, p, \alpha, \beta, T) \exp \left(\bar{\beta}|x|^{\alpha}\right) \text { for suitable } \bar{\beta}=\bar{\beta}(\alpha, \beta, p) .
\end{aligned}
$$

Therefore $I \leq C\left(I_{1}+I_{2}\right) \leq \bar{k} \exp \left(\bar{\beta}|x|^{\alpha}\right)$, and the claim is proved. (We notice that the last inequality for $I_{2}$ is true even when $\alpha=2$ if $T=T(p, \beta)$ is small.)

Now let $w=u-v$, then $w$ is c.w. upper solution of

$$
w_{t}-\Delta w=0,\left.\quad w\right|_{t=0}=0,
$$

with $|w|$ satisfying a growth condition like the one on $u$ in the statement of this lemma. Lemma 1.3 yields $w \geq 0$, i.e., $u \geq v$.

Remark 1. If $\alpha=2$, then the conclusion is still true for small $T=T(p, \beta)$. Moreover, if $u \geq 0, T$ can also be large. The proof of the second part of this remark is as follows. By the first part of this remark, there exists an $\varepsilon=$ $\varepsilon(p, \beta)>0$ such that for $t \in[0, \varepsilon]$ and $f(x, t)=|x|^{l} u^{p}(x, t) \geq 0$, we have

$$
\begin{aligned}
u(\cdot, t+\varepsilon) & \geq e^{t \Delta} u(\cdot, \varepsilon)+\int_{0}^{t} e^{(t-s) \Delta} f(\cdot, s+\varepsilon) d s \\
& \geq e^{t \Delta}\left(e^{\varepsilon \Delta} \phi+\int_{0}^{\varepsilon} e^{(\varepsilon-s) \Delta} f(\cdot, s) d s\right)+\int_{0}^{t} e^{(t-s) \Delta} f(\cdot, s+\varepsilon) d s \\
& =e^{(t+\varepsilon) \Delta} \phi+\int_{0}^{\varepsilon} e^{(t+\varepsilon-s) \Delta} f(\cdot, s) d s+\int_{\varepsilon}^{t+\varepsilon} e^{(t+\varepsilon-s) \Delta} f(\cdot, s) d s \\
& =e^{(t+\varepsilon) \Delta} \phi+\int_{0}^{t+\varepsilon} e^{(t+\varepsilon-s) \Delta} f(\cdot, s) d s .
\end{aligned}
$$

(The third step can be justified by Fubini Theorem for positive functions). Thus Lemma 1.5 is also true on $R^{n} \times[0,2 \varepsilon]$. In the same way, we can prove that Lemma 1.5 holds on $R^{n} \times[0,3 \varepsilon], \ldots, R^{n} \times[0, k \varepsilon], R^{n} \times[0, T]$.

Remark 2. Lemma 1.5 is also true for the first boundary-initial value problem with some obvious modifications.

\section{LOCAL SOLUTIONS}

In this section, we shall establish local existence of solutions for Cauchy problem (0.6) and some properties of local solutions. We first consider the 
singular case when $-2<l<0$, i.e.,

$$
\begin{aligned}
& u_{t}=\Delta u+|x|^{l} u^{p}, \quad x \in R^{n}, t>0 \\
& \left.u\right|_{t=0}=\phi \in C_{B}\left(R^{n}\right),
\end{aligned}
$$

where $p>1, n \geq 3,-2<l<0$, and $u^{p}=|u|^{p-1} u$ (to include solutions without sign condition, though we are mainly concerned with nonnegative solutions in the other sections). As was mentioned in $\S 0$, when $l<0$, it is generally impossible to obtain classical solutions for (2.1). We need the following

Definition 2.1. When $-2<l<0$, we call a function $u$ a $C_{B}$-mild solution of (2.1) on $R^{n} \times[0, T)$ if

(i) $u \in C_{B}\left(R^{n} \times\left[0, T^{\prime}\right]\right)=C\left(R^{n} \times\left[0, T^{\prime}\right]\right) \cap L^{\infty}\left(R^{n} \times\left[0, T^{\prime}\right]\right)$ for any $0<T^{\prime}<T$

(ii) $u(x, t)=\left(e^{t \Delta} \phi+\int_{0}^{t} e^{(t-s) \Delta}|\cdot|^{l} u^{p}(\cdot, s) d s\right)(x)$ for all $(x, t) \in R^{n} \times[0, T)$.

We define a $C_{B}$-mild upper (lower) solution by replacing "=" in (ii) by " $\geq$ " (“s”).

Remark. By Lemma 1.5, a continuous weak solution of (2.1) satisfying (i) of Definition 2.1 is also a $C_{B}$-mild solution. The converse of this is also true by the proof of Lemma 1.5. By the regularity theory for parabolic equations, a $C_{B}$-mild solution $u$ belongs to $C_{\mathrm{loc}}^{2,1}\left(\left(R^{n} \backslash\{0\}\right) \times(0, T)\right) \cap C_{\mathrm{loc}}^{\alpha, \alpha / 2}\left(R^{n} \times[0, T]\right)$ for any $\alpha \in(0,2+l)$. Also from Lemma 1.5 , we have

Lemma 2.2. If $u$ is a bounded and continuous weak upper (lower) solution of the elliptic equation $\Delta u+|x|^{l} u^{p}=0$ in $R^{n}(n \geq 3$ and $l>-2)$, then $u$ is a $C_{B}$-mild upper (lower) solution of (2.1) provided $\phi \leq(\geq) u$.

Now we use contraction mapping arguments to obtain local existence for (2.1) as well as some properties of local solutions of (2.1).

Theorem 2.3. (2.1) has a unique $C_{B}$-mild solution $u$ on $R^{n} \times\left[0, T_{\phi}\right)$ such that if $T_{\phi}<+\infty$, then $\lim _{t \rightarrow T_{\phi}^{-}}\|u(\cdot, t)\|_{L^{\infty}\left(R^{n}\right)}=+\infty$. Furthermore, if $\phi \geq 0$, then $u \geq 0$; if $\phi$ is radial, then $u$ is radial in $x$; if $\phi$ is radial and radially nonincreasing, then $u$ is nonincreasing in $r=|x|$.

Proof. Define $F(u)=e^{t \Delta} \phi+\int_{0}^{t} e^{(t-s) \Delta}|\cdot|^{l} u^{p}(\cdot, s) d s$. Let $B_{\delta}^{T}(\phi)$ be the closed ball in $C_{B}\left(R^{n} \times[0, T]\right)$ with center at $\phi$ and radius $\delta=3\|\phi\|_{L^{\infty}\left(R^{n}\right)}(T$ is to be chosen later). We shall show $F: B_{\delta}^{T}(\phi) \rightarrow B_{\delta}^{T}(\phi)$ and is contracting for $\delta=3\|\phi\|_{L^{\infty}\left(R^{n}\right)}$ and some $T$ depending only on $\|\phi\|_{L^{\infty}\left(R^{n}\right)}, l, n$, and $p$. Then the existence and uniqueness part of Theorem 2.1 follows.

If $u \in B_{\delta}^{T}(\phi)$, then for any $(x, t) \in R^{n} \times[0, T]$

$$
\begin{aligned}
\mid F(u) & -\phi \mid(x, t) \\
& \leq 2\|\phi\|_{L^{\infty}\left(R^{n}\right)}+\int_{0}^{t} \int_{R^{n}}(4 \pi(t-s))^{-n / 2} \exp \left(\frac{-|x-y|^{2}}{4(t-s)}\right)|y|^{l}|u|^{p}(y, s) d y d s \\
& =2\|\phi\|_{L^{\infty}\left(R^{n}\right)}+I
\end{aligned}
$$




$$
\begin{aligned}
I & \leq \int_{0}^{t} \int_{R^{n}}(4 \pi(t-s))^{-n / 2} \exp \left(\frac{-|x-y|^{2}}{4(t-s)}\right)|y|^{l} d y d s\left(4\|\phi\|_{L^{\infty}\left(R^{n}\right)}\right)^{p} \\
& \leq \int_{0}^{t} \int_{R^{n}}(4 \pi(t-s))^{-n / 2} \exp \left(\frac{-|y|^{2}}{4(t-s)}\right)|y|^{l} d y d s\left(4\|\phi\|_{L^{\infty}\left(R^{n}\right)}\right)^{p} \\
& \leq \int_{0}^{t} \int_{R^{n}} e^{-|\eta|^{2} / 4} \sqrt{(t-s)^{l}}|\eta|^{l} d \eta d s\left(4\|\phi\|_{L^{\infty}\left(R^{n}\right)}\right)^{p} \\
& \leq C(p, n, l)\|\phi\|_{L^{\infty}\left(R^{n}\right)}^{p} T^{1+l / 2} .
\end{aligned}
$$

Therefore,

$$
\|F(u)-\phi\|_{L^{\infty}\left(R^{n} \times[0, T]\right)} \leq 2\|\phi\|_{L^{\infty}\left(R^{n}\right)}+C(p, n, l)\|\phi\|_{L^{\infty}\left(R^{n}\right)}^{p} T^{1+l / 2} .
$$

Similarly, for $u$ and $v \in B_{\delta}^{T}(\phi)$, there exists $C(p, n, l)$ such that

$$
\|F(u)-F(v)\|_{L^{\infty}\left(R^{n} \times[0, T]\right)} \leq C(p, n, l)\|\phi\|_{L^{\infty}\left(R^{n}\right)}^{p-1} T^{1+l / 2}\|u-v\|_{L^{\infty}\left(R^{n} \times[0, T]\right)} .
$$

Thus we can choose a $T$ depending on $p, l, n$, and $\|\phi\|_{L^{\infty}\left(R^{n}\right)}$ such that $F: B_{T}^{\delta}(\phi) \rightarrow B_{T}^{\delta}(\phi)$ and is contracting (as shown in Claim 1 of proof of Lemma 1.5, $F(u) \in C\left(R^{n} \times[0, T]\right)$ if $\left.u \in C_{B}\left(R^{n}\right)\right)$. The proof of the first part of this theorem can be completed now by a ladder argument.

To prove the second part, we observe that it suffices to prove that $u$ satisfies those properties when $0 \leq t \leq T$ ( $T$ is chosen as in the proof above), because $t$ can go up to $T_{\phi}$ by a ladder argument. Suppose we take $\phi$ at the beginning of the iteration scheme from which the fixed point $u$ of $F$ is obtained. Since $e^{t \Delta}$ and hence $F$ preserve the properties desired (see Lemma 1.4), all terms in the iteration scheme satisfy the properties we want and hence so does the fixed point $u$ (on $R^{n} \times[0, T]$ ).

The next theorem sets up a comparison principle for (2.1), which cannot be obtained either by lemma 1.2 or Lemma 1.3 owing to the singularity in (2.1).

Theorem 2.4. (i) Suppose that $\bar{u}$ and $\underline{u}$ are $C_{B}$-mild upper and lower solutions of (2.1) on $R^{n} \times[0, T)$. Then $\bar{u} \geq \underline{u}$ on $R^{n} \times[0, T)$, and the unique $C_{B}$-mild solution of (2.1) on $R^{n} \times\left[0, T_{\phi}\right)$ satisfies that $\underline{u} \leq u \leq \bar{u}$ on $R^{n} \times[0, T)$ and $T_{\phi} \geq T$.

(ii) If the initial value $\phi$ in (2.1) is a bounded c.w. upper (lower) solution of $\Delta u+|x|^{l}|u|^{p-1} u=0$, then the $C_{B}$-mild solution $u$ of (2.1) is nonincreasing (nondecreasing) in $t \in\left[0, T_{\phi}\right)$.

Proof of (i). Let $\widetilde{T}=\min \left(T, T_{\phi}\right)$. It suffices to prove that $\underline{u} \leq u \leq \bar{u}$ on $R^{n} \times[0, \widetilde{T})$. By the proof of Theorem 2.3, there exists $T_{0}=T_{0}\left(\|\phi\|_{\infty}, p, l, n\right)$ (without loss of generality, assume $\widetilde{T}>T_{0}$ ) such that the operator

$$
F(u) \equiv e^{t \Delta} \phi+\int_{0}^{t} e^{(t-s) \Delta}|\cdot|{ }^{l} u^{p}(\cdot, s) d s
$$

maps $B_{\delta}^{T_{0}}(\phi)$ into $B_{\delta}^{T_{0}}(\phi)$ and is contracting, where $\delta=3\|\phi\|_{\infty}$. Let $\bar{B}=$ $\left\{v \in B_{\delta}^{T_{0}}(\phi) \mid v \leq \bar{u}\right\}$. For any $v \in \bar{B}, F(v) \leq F(\bar{u}) \leq \bar{u}$, i.e., $F(v) \in \bar{B}$. So $u$, which is also the fixed point of $F$ in $\bar{B}$, satisfies $u \leq \bar{u}$ on $R^{n} \times\left[0, T_{0}\right]$. Now, taking $\phi$ as $\left.u\right|_{t=T_{0}}$, we use the same reasoning to conclude that $u \leq \bar{u}$ on 
$R^{n} \times\left[T_{0}, T_{1}\right]$, where $T_{1}$ depends on $L^{\infty}$ norm of $u\left(\cdot, T_{0}\right)$ which is dominated by that of $\bar{u}\left(\cdot, T_{0}\right)$. Similarly, $u \leq \bar{u}$ on $\left[T_{1}, T_{2}\right], \ldots$. Such a ladder argument then yields $u \leq \bar{u}$ on $R^{n} \times[0, \widetilde{T})$. Similarly $\underline{u} \leq u$ on $R^{n} \times[0, \widetilde{T})$.

Proof of (ii). From Lemma 2.2, $\phi$ is also a $C_{B}$-mild upper solution of (2.1). So by (i), $u \leq \phi$ on $R^{n} \times\left[0, T_{\phi}\right)$. For any $0<h<T_{\phi}$, let $v(x, t)=u(x, t+h)$ on $R^{n} \times\left[0, T_{\phi}-h\right)$, then

$$
\begin{aligned}
v(t) & =e^{(t+h) \Delta} \phi+\int_{0}^{t+h} e^{(t+h-s) \Delta}|\cdot|^{l} u^{p}(\cdot, s) d s \\
& =e^{t \Delta}\left(e^{h \Delta}+\int_{0}^{h} e^{(h-s) \Delta}|\cdot|^{l} u^{p}(\cdot, s) d s\right)+\int_{0}^{t} e^{(t-s) \Delta}|\cdot|^{l} u^{p}(\cdot, s+h) d s \\
& =e^{t \Delta} u(h)+\int_{0}^{t} e^{(t-s) \Delta}|\cdot|^{l} v^{p}(\cdot, s) d s \\
& \leq e^{t \Delta} \phi+\int_{0}^{t} e^{(t-s) \Delta}|\cdot|^{l} v^{p}(\cdot, s) d s .
\end{aligned}
$$

So $v$ is a $C_{B}$-mild lower solution of $(2.1)$ on $R^{n} \times\left[0, T_{\phi}-h\right)$. By (i) again, $v \leq u$ on $R^{n} \times\left[0, T_{\phi}-h\right)$, i.e., $u$ is nonincreasing in $t \in\left[0, T_{\phi}\right)$.

Next, we turn to (2.1) with $l \geq 0$ (we just need $n \geq 1$ when $l \geq 0$ ).

Theorem 2.5. Suppose $l \geq 0$ in (2.1). Let $w(x)=(1+|x|)^{1 /(p-1)}$.

(i) If the initial value $\phi \in C_{w}\left(R^{n}\right)=\left\{\phi \in C\left(R^{n}\right) \mid\|\phi w\|_{L^{\infty}\left(R^{n}\right)}<+\infty\right\}$, then there exists a $T_{\phi}>0$ so that (2.1) has a unique classical solution $u$ on $R^{n} \times\left[0, T_{\phi}\right)$ so that $\|u(\cdot, t)\|_{C_{w}\left(R^{n}\right)}$ is bounded on $\left[0, T^{\prime}\right]$ for any $T^{\prime}<T_{\phi}$, and if $T_{\phi}<+\infty$, then $\lim _{t \rightarrow T_{\phi}^{-}}\|u(\cdot, t)\|_{C_{w}\left(R^{n}\right)}=+\infty$.

(ii) If $\phi \in C\left(R^{n}\right), \liminf _{|x| \rightarrow+\infty}|x|^{a} \phi(x)>0$ for some $a=1 /(p-1)-$ $\varepsilon(\varepsilon>0)$, then there exists no $T>0$ such that $(2.1)$ has a classical solution $u$ on $R^{n} \times[0, T]$ with $0 \leq u(x, t) \leq C(T)(1+|x|)^{m}$ on $R^{n} \times[0, T]$ for some $m>0$.

Proof of (i). The uniqueness is a simple consequence of Lemma 1.3. For the existence, let $X=L_{w}^{\infty}\left(R^{n}\right)=\left\{\phi \mid\|\phi w\|_{L^{\infty}\left(R^{n}\right)}<+\infty\right\}$. We claim that there exists a $T_{\phi}>0$ such that the integral equation

$$
u=e^{t \Delta} \phi+\int_{0}^{t} e^{(t-s) \Delta}|\cdot|^{l} u^{p}(\cdot, s) d s
$$

has a solution $u \in L_{\text {loc }}^{\infty}\left(\left[0, T_{\phi}\right), X\right)$ satisfying that if $T_{\phi}<+\infty$, then $\|u(\cdot, t)\|_{X}$ $\rightarrow+\infty$ as $t \rightarrow T_{\phi}^{-}$. From this assertion and by the proof of Lemma 1.5, $u$ is a c.w. solution of (2.2) satisfying the properties in the statement of (i). Then (i) follows from the fact that $u$ is also a classical solution, which is a consequence of the regularity theory for parabolic equations. The proof of the claim is as follows. Let

$$
B_{\delta}^{T}(\phi)=\left\{u \in L^{\infty}([0, T], X) \mid\|u(\cdot, t)-\phi\|_{X} \leq \delta \text { for } 0 \leq t \leq T\right\} .
$$

$\delta$ and $T$ are to be chosen so that $F(F(u)$ is defined as the right-hand side of (2.2)) satisfies $F: B_{\delta}^{T}(\phi) \rightarrow B_{\delta}^{T}(\phi)$ and is contracting. For any $u \in B_{\delta}^{T}$ and 
$0 \leq t \leq T$,

$$
\begin{aligned}
&|F(u)-\phi|(x, t) \leq \frac{1}{(4 \pi t)^{n / 2}} \int_{R^{n}} \exp \left(\frac{-|x-y|^{2}}{4 t}\right)|\phi(y)| d y+|\phi(x)| \\
& \quad \int_{0}^{t} \int_{R^{n}}(4 \pi(t-s))^{-n / 2} \exp \left(\frac{-|x-y|^{2}}{4(t-s)}\right)|y|^{l}|u|^{p}(y, s) d y d s \\
&= I_{1}+|\phi(x)|+I_{2} \\
& \leq I_{1}+\|\phi\|_{X}(w(x))^{-1}+I_{2} . \\
& I_{1} \leq(4 \pi t)^{-n / 2} \int_{R^{n}} \exp \left(\frac{-|x-y|^{2}}{4 t}\right)\|\phi\|_{X}(1+|y|)^{-l /(p-1)} d y \\
&=C\|\phi\|_{X} \int_{R^{n}} \exp \left(\frac{-|\eta|^{2}}{4}\right)(1+\mid x-\sqrt{t} \eta)^{-l /(p-1)} d \eta \\
&=C\|\phi\|_{X}\left(\int_{R_{1}}+\int_{R_{2}}\right) \exp \left(\frac{-|\eta|^{2}}{4}\right)(1+\mid x-\sqrt{t} \eta)^{-l /(p-1)} d \eta \\
& \quad\left(R_{1}=\{\eta|| x-\sqrt{t} \eta|\leq| x \mid / 2\}, R_{2}=\{\eta|| x-\sqrt{t} \eta|\geq| x \mid / 2\}\right) \\
& \leq C\|\phi\|_{X}\left(\int_{R_{1}} e^{-|\eta|^{2} / 4} d \eta+\left(1+\frac{|x|}{2}\right)^{-l /(p-1)} \int_{R^{n}} e^{-|\eta|^{2} / 4} d \eta\right) \\
& \leq C\|\phi\|_{X}\left(\int_{|x| / 2 \sqrt{t}}^{+\infty} e^{-r^{2} / 4} r^{n-1} d r+w^{-1}(x)\right) \\
& \leq C\|\phi\|_{X}\left(\int_{|x| / 2 \sqrt{t}}^{+\infty} e^{-r^{2} / 8} r d r+w^{-1}(x)\right) \\
& \leq C\|\phi\|_{X}\left(e^{-|x|^{2} / 32 t}+w^{-1}(x)\right) \\
& \leq C(p, n, l)\|\phi\|_{X} w^{-1}(x)
\end{aligned}
$$

(without loss of generality, assume $T>1$ );

$$
\begin{aligned}
I_{2} & \leq \int_{0}^{t} \int_{R^{n}}(4 \pi(t-s))^{-n / 2} \exp \left(\frac{-|x-y|^{2}}{4(t-s)}\right)|y|^{l}\|u(\cdot, s)\|_{X}^{p} w^{-p}(y) d y d s \\
& \leq \int_{0}^{t} \int_{R^{n}}(4 \pi(t-s))^{-n / 2} \exp \left(\frac{-|x-y|^{2}}{4(t-s)}\right)(1+|y|)^{-l /(p-1)} d y d s\left(\|\phi\|_{X}^{p}+\delta^{p}\right) \\
& \left.\leq C \int_{0}^{t}\left(e^{-|x|^{2} / 32 s}+w^{-1}(x)\right) d s\left(\|\phi\|_{X}^{p}+\delta^{p}\right) \quad \text { just as for } I_{1}\right) \\
& \leq C(p, n, l)\left(\|\phi\|_{X}^{p}+\delta^{p}\right) t w^{-1}(x) .
\end{aligned}
$$

Now returning to the estimate of $|F(u)-\phi|$, we have

$$
\|F(u)-\phi\|_{X}(t) \leq C_{1}(p, n, l)\left(\|\phi\|_{X}+T\left(\|\phi\|_{X}^{p}+\delta^{p}\right)\right) .
$$

Similarly, for any $u$ and $v \in B_{\delta}^{T}(\phi)$,

$$
\|F(u)-F(v)\|_{X}(t) \leq C_{2}(p, n, l) T\left(\|\phi\|_{X}^{p-1}+\delta^{p-1}\right)\|u-v\|_{X}(t) .
$$

Let $\delta=2 C_{1}(p, n, l)\|\phi\|_{X} \quad\left(C_{1}\right.$ is as in (2.3)), and take $T=T\left(p, n, l,\|\phi\|_{X}\right)<$ 1 such that the right-hand side of $(2.3) \leq \delta$ and the coefficient of $\|u-v\|_{X}$ on the right-hand side of $(2.4)<1$. These, (2.3), and (2.4) then imply that 
$F(u)$ has a (unique) fixed point in $B_{\delta}^{T}(\phi)$. A ladder argument then completes the proof of the claim concerning (2.2) and (i) is proved.

Proof of (ii). Suppose that there exists $T>0$ such that (2.1) has a classical solution $u$ on $R^{n} \times[0, T]$ with $0 \leq u(x, t) \leq C(T)(1+|x|)^{m}$ on $R^{n} \times[0, T]$ for some $m>0$. Without loss of generality, assume $T=1$. By Lemma 1.5, for $(x, t) \in R^{n} \times[0,1]$,

$$
u(x, t)=\left(e^{t \Delta} \phi+\int_{0}^{t} e^{(t-s) \Delta}|\cdot|^{l} u^{p}(\cdot, s) d s\right)(x) .
$$

Since $u \geq 0$, we have for $(x, t) \in R^{n} \times[0,1]$,

$$
\begin{aligned}
u(x, t) & \geq\left(e^{t \Delta} \phi\right)(x) \\
& \geq C t^{-n / 2} \int_{R \leq|y|} \exp \left(\frac{-|x-y|^{2}}{4 t}\right)|y|^{-a} d y \quad\left(\liminf _{|x| \rightarrow+\infty}|x|^{a} \phi(x)>0\right) \\
& \geq C t^{-n / 2} \int_{|x-y| \leq \sqrt{t}} \exp \left(\frac{-|x-y|^{2}}{4 t}\right)|y|^{-a} d y \quad \text { if }|x| \geq R+1 .
\end{aligned}
$$

Observe that when $|x-y| \leq \sqrt{t},|x|-\sqrt{t} \leq|y| \leq|x|+\sqrt{t}$. So if $|x| \geq R+1$

$$
\begin{aligned}
u(x, t) & \geq C t^{-n / 2} \int_{|x-y| \leq \sqrt{t}} \exp \left(\frac{-|x-y|^{2}}{4 t}\right)(1+|x|)^{-a} d y \\
& =C(\phi, n)(1+|x|)^{-a} .
\end{aligned}
$$

Let $B_{1}(x, t)=|x|^{l} u^{p}(x, t)$. By the lower bound of $u$ obtained above,

$$
B_{1}(x, t) \geq C(n, \phi, p)(1+|x|)^{-a p+l} \text { for }|x| \geq R+1, t \in[0,1] .
$$

Let $a_{1}=-(-a p+l)=l /(p-1)-\varepsilon p$. Then as above, we have for $|x| \geq R+2$

$$
\begin{aligned}
u(x, t) & \geq \int_{0}^{t} e^{(t-s) \Delta} B_{1}(\cdot, s) d s \\
& \geq \int_{0}^{t} C(n, \phi, p)(1+|x|)^{-a_{1}} d s \\
& \geq t C(n, \phi, p)(1+|x|)^{-a_{1}} .
\end{aligned}
$$

Take a small $\delta>0$. Then for $|x| \geq R+2$ and $\delta \leq t \leq 1$, we have $u(x, t) \geq$ $\delta C(n, \phi, p)(1+|x|)^{-a_{1}}$. Note by Lemma 1.5 again, on $R^{n} \times[0,1-\delta]$

$$
u(x, t+\delta)=\left(e^{t \Delta} u(\cdot, \delta)+\int_{0}^{t} e^{(t-s) \Delta}|\cdot|^{l} u^{p}(\cdot, s+\delta) d s\right)(x),
$$

especially

$$
u(x, t+\delta) \geq \int_{0}^{t} e^{(t-s) \Delta}|\cdot|^{l} u^{p}(\cdot, s+\delta) d s .
$$

Thus the same argument as above implies that for $|x| \geq R+3$ and $2 \delta \leq t \leq 1$, $u(x, t) \geq C(n, \phi, \delta, p)(1+|x|)^{-a_{2}}$, where $a_{2}=-\left(-a_{1} p+l\right)=l /(p-1)-\varepsilon p^{2}$. In such a way, for any integer $0<k<1 / \delta$, we have

$$
u(x, t) \geq C(n, \phi, \delta, p, k)(1+|x|)^{-a_{k}}
$$

for $|x| \geq R+C(k)$ and $t \in[k \delta, 1]$, where $a_{k}=l /(p-1)-\varepsilon p^{k}$. This contradicts the growth condition on $u$. 
Remark 1. By simple modifications of the proof above, Theorem 2.4 remains true for the case $-2<l<0$. In particular, when $-2<l<0,(2.1)$ still has a c.w. solution even when $\phi$ has a certain growth. But in this paper we would like to concentrate only on bounded solutions and hence, not pursuing the greatest generality, we prefer $C_{B}$-mild solutions.

Remark 2. The local classical solution of $(2.1)$ with $l \geq 0$ is unique with respect to the more general class consisting of all smooth $u$ such that $|u(x, t)| \leq$ $K(1+|x|)^{(2-l) /(p-1)}$, by the Phragmen-Lindelöf principle (see Lemma 1.3).

Lemma 2.6. Consider (2.1) with $l \geq 0$. Suppose that $u$ is a classical solution on $R^{n} \times[0, T)$ with $|u(x, t)| \leq K\left(T^{\prime}\right)(1+|x|)^{(2-l) /(p-1)}$ on $R^{n} \times\left[0, T^{\prime}\right]$ for any $0<T^{\prime}<T$. Then the following statements hold.

(i) If the initial value $\phi$ is radial, then $u$ is radial in $x$.

(ii) If $\phi$ is a c.w. upper (lower) solution but not a solution of $\Delta u+|x|^{l}|u|^{p-1} u=$ 0 , then $u_{t}(x, t)<(>) 0, t>0$.

Proof. (i) can be easily proved by the reflection argument as in [13], we omit the detail. To prove (ii), using Phragmèn-Lindelöf principle (see Lemma 1.3), we have $\phi \geq u$. For a small $h>0$, let $u_{h}(x, t)=u(x, t+h), w=u-u_{h}$. Then $\left.w\right|_{t=0}=\phi-u(\cdot, h) \geq 0, w_{t}-\Delta w=|x|^{l}\left(u^{p}-u_{h}^{p}\right)=C(x, t) w$, where $C(x, t) \leq C_{0}\left(T^{\prime}\right)\left(1+|x|^{2}\right)$ on $R^{n} \times\left[0, T^{\prime}\right]$. Therefore, by Lemma 1.3 again, $w \geq 0$, i.e., $u$ is nonincreasing in $t$. Hence $u_{t} \leq 0$ if $t>0$. Now (ii) follows from the strong maximum principle.

\section{Global EXISTENCE AND Finite time Blow-UP}

We begin with a necessary condition for existence of nonnegative global solutions of

$$
\begin{aligned}
& u_{t}=\Delta u+|x|^{l} u^{p}, \quad x \in R^{n}, \quad t>0, u \geq 0, \\
& \left.u\right|_{t=0}=\phi \in C\left(R^{n}\right), \quad \phi \geq 0, \quad \phi \not \equiv 0,
\end{aligned}
$$

where $p>1, l>-2$, and $n \geq 3$.

For a bounded domain $\Omega$ in $R^{n}$, let $\lambda(\Omega)$ be the first eigenvalue of $-\Delta$ with zero boundary condition, and let $\psi_{\Omega}$ be the corresponding eigenfunction with $\int_{\Omega} \psi_{\Omega}=1$. Let $B_{R}=\{|x|<R\}$ and $\Omega_{R}=\{R<|x|<2 R\}$. Following the idea of Lee and $\mathrm{Ni}$ [24], we have

Proposition 3.1. If (3.1) has a (nonnegative) global c.w. solution $u$, then

(i) when $l>0$,

$$
\int_{\Omega_{R}} u(x, t) \psi_{\Omega_{R}}(x) d s \leq\left(R^{-(2+l)} \lambda\left(\Omega_{1}\right)\right)^{1 /(p-1)}
$$

and

$$
\liminf _{|x| \rightarrow+\infty}|x|^{(2+l) /(p-1)} u(x, t) \leq 2^{(2+l) /(p-1)}\left(\lambda\left(\Omega_{1}\right)\right)^{1 /(p-1)}
$$

for all $t \geq 0$;

(ii) when $-2<l \leq 0$,

$$
\int_{B_{R}} u(x, t) \psi_{B_{R}}(x) \leq\left(R^{-(2+l)} \lambda\left(B_{1}\right)\right)^{1 /(p-1)}
$$


and

$$
\liminf _{|x| \rightarrow+\infty}|x|^{(2+l) /(p-1)} u(x, t) \leq\left(\lambda\left(B_{1}\right)\right)^{1 /(p-1)}
$$

for all $t \geq 0$.

Remark. Proposition 3.1 applies to $C_{B}$-mild solutions because a $C_{B}$-mild solution is also a c.w. solution.

Proof of Proposition 3.1. To prove (i), we notice that by regularity theory of parabolic equation, $u$ is classical when $l \geq 0$. Multiplying $\psi_{\Omega_{R}}$ to the differential equation in (3.1) and integrating over $\Omega_{R}$, we have by Jensen's inequality

$$
F_{R}^{\prime}(t) \geq-\lambda\left(\Omega_{R}\right) F_{R}(t)+R^{l} F_{R}^{p}(t), \quad t>0,
$$

where

$$
F_{R}(t)=\int_{\Omega_{R}} u(x, t) \psi_{\Omega_{R}}(x) d x .
$$

If there exists $t_{0} \geq 0$ such that $-\lambda\left(\Omega_{R}\right) F_{R}\left(t_{0}\right)+R^{l} F_{R}^{p}\left(t_{0}\right)>0$, then by (3.2), $F_{R}(t)$ ultimately increasingly $\rightarrow+\infty$ as $t \rightarrow+\infty$. Therefore there exists $c>0$ and $t_{1}>0$ such that $F_{R}^{\prime}(t) \geq c F_{R}^{p}(t)$ if $t \geq t_{1}$, hence

$$
\int_{F_{R}\left(t_{1}\right)}^{F_{R}(t)} \frac{d F}{F^{p}} \geq c\left(t-t_{1}\right) \quad \text { if } t \geq t_{1} .
$$

This is impossible. Therefore for all $t \geq 0,-\lambda\left(\Omega_{R}\right)+R^{l} F_{R}^{p-1}(t) \leq 0$, i.e.,

$$
\int_{\Omega_{R}} u \psi_{\Omega_{R}}=F_{R}(t) \leq\left(R^{-l} \lambda\left(\Omega_{R}\right)\right)^{1 /(p-1)} .
$$

Since $\lambda\left(\Omega_{R}\right)=R^{-2} \lambda\left(\Omega_{1}\right)$, we then have

$$
\int_{\Omega_{R}} u(x, t) \psi_{\Omega_{R}}(x) d x \leq\left(R^{-(2+l)} \lambda\left(\Omega_{1}\right)\right)^{1 /(p-1)} .
$$

To prove the remainder of (i), we observe

$$
\begin{aligned}
& \int_{\Omega_{R}}|x|^{(2+l) /(p-1)} u(x, t) \psi_{R}(x) d x \\
& \quad \leq(2 R)^{(2+l) /(p-1)} \int_{\Omega_{R}} u(x, t) \psi_{R}(x) d x \leq 2^{(2+l) /(p-1)}\left(\lambda\left(\Omega_{1}\right)\right)^{1 /(p-1)} .
\end{aligned}
$$

Thus

$$
\begin{aligned}
& \inf _{R \leq|x| \leq 2 R}|x|^{(2+l) /(p-1)} u(x, t) \\
& \quad \leq \int_{\Omega_{R}}|x|^{(2+l) /(p-1)} u(x, t) \psi_{R}(x) d x \leq 2^{(2+l) /(p-1)}\left(\lambda\left(\Omega_{1}\right)\right)^{1 /(p-1)}
\end{aligned}
$$

and hence

$$
\liminf _{|x| \rightarrow+\infty}|x|^{(2+l) /(p-1)} u(x, t) \leq 2^{(2+l) /(p-1)}\left(\lambda\left(\Omega_{1}\right)\right)^{1 /(p-1)} .
$$

To prove (ii), we notice that by a standard manipulation,

$$
\begin{aligned}
\left.\int_{B_{R}} u(x, s) \psi_{B_{R}}(x) d x\right|_{0} ^{t}= & \int_{0}^{t} \int_{B_{R}} u \Delta \psi_{B_{R}}+|x|^{l} u^{p} \psi_{B_{R}} d x d s \\
& -\int_{0}^{t} \int_{\partial B_{R}} u(x, s) \frac{\partial \psi_{R}(x)}{\partial \eta} d \sigma d s
\end{aligned}
$$


It is easy to see that the right-hand side of (3.3) is differentiable in $t$. Then so is the left-hand side and

$$
\begin{aligned}
\frac{d}{d t} F_{R}(t) & \equiv \frac{d}{d t} \int_{B_{R}} u(x, t) \psi_{B_{R}}(x) d x \\
& \geq \int_{B_{R}} u(x, t) \Delta \psi_{B_{R}}(x)+|x|^{l} u^{p}(x, t) \psi_{B_{R}}(x) d x
\end{aligned}
$$

From this and by the same argument in the proof of (i), we have

$$
\int_{B_{R}} u(x, t) \psi_{B_{R}}(x) d x \leq\left(R^{-(2+l)} \lambda\left(B_{1}\right)\right)^{1 /(p-1)} .
$$

To prove the second part of (ii), we notice $\psi_{B_{R}}(x)=\left(1 / R^{n}\right) \psi_{B_{1}}(x / R)$, so from the above inequality we have

$$
\int_{B_{1}} u(R x, t) \psi_{B_{1}}(x) d x \leq R^{-(2+l) /(p-1)}\left(\lambda\left(B_{1}\right)\right)^{1 /(p-1)},
$$

hence

$$
\begin{gathered}
\int_{B_{1}}|R x|^{(2+l) /(p-1)} u(R x, t) \psi_{B_{1}}(x) d x \leq\left(\lambda\left(B_{1}\right)\right)^{1 /(p-1)}, \\
\inf _{\varepsilon R \leq|x| \leq R}|x|^{(2+l) /(p-1)} u(x, t) \int_{B_{1}-B_{\varepsilon}} \psi_{B_{1}}(x) d x \leq\left(\lambda\left(B_{1}\right)\right)^{1 /(p-1)} .
\end{gathered}
$$

Letting $R \rightarrow+\infty$ and then $\varepsilon \rightarrow 0^{+}$, we obtain the result desired.

Corollary 3.2. For any sequence $t_{m} \rightarrow+\infty$, denote $\lim \inf _{m \rightarrow+\infty} u\left(x, t_{m}\right)$ by $u_{\infty}(x)$, then the conclusion of Proposition 3.1 is true for $u_{\infty}$. In particular, $u_{\infty} \in L_{\text {loc }}^{1}\left(R^{n} \backslash\{0\}\right)$ when $l>0, u_{\infty} \in L_{\text {loc }}^{1}\left(R^{n}\right)$ when $-2<l \leq 0$.

This corollary follows from the proof of Proposition 3.1 and Fatou's Lemma.

Next, we recall some results of positive radial solutions of

$$
\Delta u+|x|^{l} u^{p}=0 \text { in } R^{n}, l>-2, n \geq 3 \text {, and } u>0 \text {, }
$$

and of the Dirichlet boundary problem

$$
\begin{aligned}
& \Delta u+|x|^{l} u^{p}=0 \quad \text { on } B_{R}, l>-2, n \geq 3, \text { and } u>0, \\
& \left.u\right|_{\partial B_{R}}=0 .
\end{aligned}
$$

Definition 3.3. We say that $u$ is a regular solution of $(3.4)$ (or $(3.5)_{R}$ ) if $u \in$ $C^{2}\left(R^{n} \backslash\{0\}\right) \cap C\left(R^{n}\right)$ (or $u \in C^{2}\left(\bar{B}_{R} \backslash\{0\}\right) \cap C\left(\bar{B}_{R}\right)$ ) and $u$ satisfies (3.4) (or $\left.(3.5)_{R}\right)$ when $x \neq 0$. We call $u$ a singular solution of $(3.4)$ (or $\left.(3.5)_{R}\right)$ if $u \in C^{2}\left(R^{n} \backslash\{0\}\right)$ (or $u \in C^{2}\left(\bar{B}_{R} \backslash\{0\}\right)$ ) satisfies (3.4) $\left((3.5)_{R}\right)$ in $R^{n} \backslash\{0\}$ (or $\left.B_{R} \backslash\{0\}\right)$ with nonremovable singularity at $x=0$.

Proposition 3.4. (i) When $p>(n+l) /(n-2), u_{s}(r)=L r^{-(2+l) /(p-1)}$ is a singular solution of (3.4) ( $L$ is as in (0.7)).

(ii) When $(n+l) /(n-2)<p<(n+2+2 l) /(n-2)$, for each $R>0,(3.5)_{R}$ has a unique radial regular solution $u_{R}^{D} \not \equiv 0$ and $u_{R}^{D}(r)=R^{-(2+l) /(p-1)} u_{1}^{D}(r / R)$ on $[0, R], u_{R}^{D}(r)$ is decreasing in $r \in[0, R]$. Furthermore, (3.4) has no nontrivial radial regular solution. The set of all radial singular solutions of (3.4) consists of $u_{s}$ and a family of singular solutions $\left\{U_{s}\right\}$ with decay like $r^{-(n-2)}$ at $r=+\infty$ and $U_{s}(r) / u_{s}(r) \rightarrow 1$ as $r \rightarrow 0$. 
(iii) When $p=(n+2+2 l) /(n-2)$, all nontrivial regular solutions of (3.4) are of the form

$$
u_{\lambda}^{E}(r)=\left(\frac{\lambda \sqrt{(n+l)(n-2)}}{\lambda^{2}+r^{2+l}}\right)^{(n-2) /(2+l)}, \quad \lambda>0 .
$$

The set of all radial singular solutions of (3.4) consists of $u_{s}$ and a family of singular solutions oscillating around $u_{s}$ near $r=0,+\infty$.

(iv) When $p>(n+2+2 l) /(n-2)$, all nontrivial radial regular solutions of (3.4) are included in a family $\left\{u_{\alpha}\right\}_{\alpha>0}$ with $u_{\alpha}$ decreasing in $r, r^{(2+l) /(p-1)} u_{\alpha}(r)$ $\rightarrow L$ as $r \rightarrow+\infty, u_{\alpha}(0)=\alpha^{(2+l) /(p-1)}$, and $u_{\alpha}(r)=\alpha^{(2+l) /(p-1)} u_{1}(\alpha r)$. The only radial singular solution of (3.4) is $u_{s}$.

Remark 1. The above results about $(3.5)_{R}$ can be found in [30], the results about (3.4) are listed in Appendix A of [15] and can be proved by phase plane analysis.

Remark 2. All solutions above (regular or singular) are distributional solutions. This can be proved by the argument in the proof of Proposition 3.1 in [14].

Remark 3. When $1<p \leq(n+l) /(n-2)$, by Theorem 3.41 in [28], (3.4) has no nontrivial solution even in exterior domains. We should point out that when $1<p \leq(n+l) /(n-2), u_{R}^{D}$ in (ii) still exists (see [30]).

The next proposition will be useful in our analysis and may be of independent interest.

Proposition 3.5. (i) When $(n+l) /(n-2)<p<p_{c}\left(p_{c}\right.$ is as in $\left.(0.7)\right)$, if $U \not \equiv u$ are two positive radial singular (regular) solutions of $\Delta u+|x|^{l} u^{p}=0$ on $\overline{B_{1}}\left(B_{1}^{c}\right)$ with $r^{(2+l) /(p-1)} U(r) \rightarrow L, r^{(2+l) /(p-1)} u(r) \rightarrow L$ as $r \rightarrow 0^{+}(r \rightarrow+\infty) \quad(L$ is as in (0.7)), then $U$ oscillates around $u$.

(ii) When $(n+2+2 l) /(n-2)<p<p_{c}$, assume $\bar{u}(\underline{u})$ is a radial regular upper (lower) solution of (3.4). If $u_{\alpha}$ is a positive radial regular solution of (3.4) such that $\bar{u} \geq u_{\alpha}\left(\underline{u} \leq u_{\alpha}\right)$, then $u_{\alpha} \equiv \bar{u}(\underline{u})$.

(iii) When $p \geq p_{c}$, for any $\lambda>(<) 1, \bar{u}(\underline{u})$ and $u_{\alpha}$ as in (ii), then $\bar{u}(\underline{u})$ cannot stay above (below) $\lambda u_{\alpha}$.

Remark. (ii) is also true when $p=(n+2+2 l) /(n-2)$. This can be proved either by an elliptic argument or by Theorem 3.6 and Theorem 3.10. Furthermore, we note that when $p \geq p_{c}$, two radial regular solutions of (3.4) can never intersect (see Proposition 3.7).

Proof of (i) of Proposition 3.5. Let $v(t)=U(r) / u(r), t=\log r$. Then

$$
v^{\prime \prime}(t)+\left(\frac{2 r u^{\prime}(r)}{u(r)}+(n-2)\right) v^{\prime}(t)+r^{2+l} u^{p-1}(r)\left(v^{p}-v\right)(t)=0, \quad t \leq 0
$$

and $\lim _{t \rightarrow-\infty} v(t)=1$. Since $r^{(2+l) /(p-1)} u(r) \rightarrow L$ as $r \rightarrow 0^{+}, r^{2+l} u^{p-1}(r) \rightarrow$ $L^{p-1}$ as $r \rightarrow 0^{+}$or $t \rightarrow-\infty$.

To find $\lim _{r \rightarrow 0^{+}} 2 r u^{\prime}(r) / u(r)$, we define $w_{1}(t)=u(r) / u_{s}(r), t=\log r \quad\left(u_{s}\right.$ is given in Proposition 3.4). Then $w_{1}(t) \rightarrow 1$ as $t \rightarrow-\infty$ and

$$
w_{1}^{\prime \prime}+c_{0} w_{1}^{\prime}+L^{p-1}\left(w_{1}^{p}-w_{1}\right)=0 \text { on }(-\infty, 0],
$$


where

$$
c_{0}=\frac{n-2}{p-1}\left(p-\frac{n+2+2 l}{n-2}\right) .
$$

We claim that $w_{1}^{\prime} \rightarrow 0$ as $t \rightarrow-\infty$ (the following proof of this assertion is inspired by [29]).

Case 1. $p>(n+2+2 l) /(n-2)$. In this case $c_{0}$ in $(3.7)>0$. Multiplying (3.7) by $w_{1}^{\prime}$ and integrating over $[t, 0]$, we have

$$
\frac{1}{2}\left(w_{1}^{\prime}(t)\right)^{2}+c_{0} \int_{t}^{0}\left(w_{1}^{\prime}\right)^{2}=O(1) .
$$

Thus $w_{1}^{\prime}$ is bounded and $\int_{-\infty}^{0}\left(w_{1}^{\prime}\right)^{2}<+\infty$. From this and (3.7), $w_{1}^{\prime \prime}$ is bounded and hence $w_{1}^{\prime} \rightarrow 0$ as $t \rightarrow-\infty$.

Case 2. $p=(n+2+2 l) /(n-2)$. In this case $c_{0}$ in $(3.7)=0$. Similarly as in Case 1, we have for $T_{1}<T_{2}<0$

$$
\left.\frac{1}{2}\left(w_{1}^{\prime}\right)^{2}\right|_{T_{1}} ^{T_{2}}+\int_{w_{1}\left(T_{1}\right)}^{w_{1}\left(T_{2}\right)} L^{p-1}\left(w^{p}-w\right) d w=0
$$

This and the fact that $w_{1} \rightarrow 1$ as $t \rightarrow-\infty$ imply $\left(w_{1}^{\prime}\right)^{2}(t) \rightarrow 0$ as $t \rightarrow-\infty$.

Case 3. $(n+l) /(n-2)<p<(n+2+2 l) /(n-2)$. In this case $c_{0}<0$ in (3.7). It is easy to see that both $w_{1}^{\prime}$ and $w_{1}^{\prime \prime}$ must be bounded. By (3.8), $\int_{-\infty}^{0}\left(w_{1}^{\prime}\right)^{2}<+\infty$. Thus $w_{1}^{\prime} \rightarrow 0$ as $t \rightarrow-\infty$.

Now the proof of the claim is complete. From the claim, it follows that

$$
w_{1}^{\prime}(t)=\frac{1}{L}\left(\frac{2+l}{p-1} r^{(2+l) /(p-1)} u(r)+r^{(2+l) /(p-1)+1} u^{\prime}(r)\right) \rightarrow 0 \text { as } r \rightarrow 0^{+} \text {. }
$$

Thus

$$
\frac{2 r u^{\prime}(r)}{u(r)}=\frac{2 r^{(2+l) /(p-1)+1} u^{\prime}(r)}{r^{(2+l) /(p-1)} u(r)} \rightarrow \frac{-2(2+l)}{p-1} \quad \text { as } r \rightarrow 0^{+} .
$$

Let $w=v-1$. Then by (3.6) and the discussion above

$$
w^{\prime \prime}+f(t) w^{\prime}+g(t) w=0 \text { on }(-\infty, 0]
$$

with

$$
f(t) \rightarrow c_{0} \quad \text { and } \quad g(t)=r^{2+l} u^{p-1}(r) \frac{v^{p}-v}{v-1} \rightarrow(p-1) L^{P-1} \quad \text { as } t \rightarrow-\infty .
$$

(If there exist $t_{m} \rightarrow-\infty$ such that $v\left(t_{m}\right)=1$, then we are done. So we assume $v(t) \neq 1$ for large $t$ and hence $g(t)$ is well defined.)

By direct calculation, when $(n+l) /(n-2)<p<p_{c}$

$$
\left(\lim _{t \rightarrow-\infty} f(t)\right)^{2}-4\left(\lim _{t \rightarrow-\infty} g(t)\right)=c_{0}^{2}-4(p-1) L^{p-1}<0 .
$$

From this and Sturm-type arguments (or see the proof of (ii), we conclude that $w$ oscillates around 0 near $t=-\infty$ and (i) of this proposition follows.

Proof of (ii). Suppose that $\bar{u} \geq u_{\alpha}$ and $\bar{u} \not \equiv u_{\alpha}$. Let $v(t)=\bar{u}(r) / u_{\alpha}(r), t=$ $\log r$, then $v \geq 1$ and

$$
v^{\prime \prime}+\left(\frac{2 r u_{\alpha}^{\prime}(r)}{u_{\alpha}(r)}+(n-2)\right) v^{\prime}(t)+r^{2+l} u_{\alpha}^{p-1}(r)\left(v^{p}-v\right) \leq 0 \quad \text { on }(-\infty,+\infty)
$$


Denote the coefficient of $v^{\prime}$ by $f(t)$. Exactly like in the proof of (i), we have $f(t) \rightarrow c_{0}$ as $t \rightarrow+\infty$ (recall from Proposition 3.4, $r^{(2+l) /(p-1)} u_{\alpha}(r) \rightarrow L$ as $r \rightarrow+\infty$, so the argument there can go through.)

We claim $\lim _{t \rightarrow+\infty} v(t)=1$. In fact, by (3.9) and the fact $v \geq 1, v^{\prime \prime}+$ $f(t) v^{\prime} \leq 0$. Hence

$$
\exp \left(\int_{0}^{t} f(s) d s\right) v^{\prime}(t) \leq \exp \left(\int_{0}^{\tau} f(s) d s\right) v^{\prime}(\tau) \text { if } t \geq \tau .
$$

By the proof of (iii) of Proposition 4.4 in [30], $r u_{\alpha}^{\prime}(r) \rightarrow 0$ as $r \rightarrow 0^{+}$, hence $f(t) \rightarrow n-2$ as $t \rightarrow-\infty$. Since $v(t) \rightarrow \bar{u}(0) / u_{\alpha}(0)$ as $t \rightarrow-\infty$, there exists a sequence $t_{m} \rightarrow-\infty$ such that $v^{\prime}\left(t_{m}\right) \rightarrow 0$. Now in (3.10), letting $\tau=t_{m} \rightarrow-\infty$, we have either $v^{\prime}<0$ on $(-\infty,+\infty)$ or $v^{\prime} \equiv 0$. (A priori, $v^{\prime} \leq 0$ and if there exists $t_{0}$ such that $v^{\prime}\left(t_{0}\right)<0$, then by (3.10) again $v^{\prime}(t)<0$ if $t \geq t_{0}$. So $v>1$ and hence the strict inequality in (3.10) must be true which in turn implies that $v^{\prime}<0$ on $(-\infty,+\infty)$.) But $v^{\prime} \equiv 0$ is impossible because $\bar{u} \not \equiv u_{\alpha}$. Suppose $\lim _{t \rightarrow+\infty} v(t)>1$, then by (3.9) and the fact $v^{\prime}<0$, we have for a large $T$ and some constant $c>0$

$$
v^{\prime \prime}+f(t) v^{\prime} \leq-c \text { if } t \geq T .
$$

This forces $v=0$ at some $t$. Thus we finish the proof of $\lim _{t \rightarrow+\infty} v(t)=1$ (and $v>1, v^{\prime}<0$ ).

Now let $w=v-1>0$. By (3.9) and the discussion above, we have

$$
w^{\prime \prime}+f(t) w^{\prime}+g(t) w \leq 0, \quad w^{\prime}<0 \quad \text { on }(-\infty,+\infty),
$$

with $f(t) \rightarrow c_{0}, g(t)=r^{2+l} u_{\alpha}^{p-1}(r)\left(v^{p}-v\right) /(v-1) \rightarrow(p-1) L^{p-1}$ as $t \rightarrow+\infty$. As before, when $(n+l) /(n-2)<p<p_{c}$,

$$
\left(\lim _{t \rightarrow+\infty} f(t)\right)^{2}-4\left(\lim _{t \rightarrow+\infty} g(t)\right)<0 .
$$

Then there exist $T>0, b_{1}$ and $c_{1}$ such that $b_{1}^{2}-4 c_{1}<0, f(t)<b_{1}$, and $g(t)>c_{1}$ if $t \geq T$. Observe that any solution of

$$
W^{\prime \prime}+b_{1} W^{\prime}+c_{1} W=0
$$

is oscillatory; in particular, there exist $b>a>T$ such that $W(a)=W(b)=0$, $W>0$ on $(a, b)$ (and hence $W^{\prime}(a)>0>W^{\prime}(b)$ ). Multiplying (3.11) by $W$ and (3.12) by $w$, we have

$$
\begin{gathered}
w^{\prime \prime} W+f(t) w^{\prime} W+g(t) w W \leq 0 \quad \text { on }[a, b], \\
W^{\prime \prime} w+b_{1} W^{\prime} w+c_{1} w W=0 \quad \text { on }[a, b] .
\end{gathered}
$$

Subtracting (3.14) from (3.13) yields

$$
\left(W w^{\prime}-W^{\prime} w\right)^{\prime}+\left(f(t) w^{\prime} W-b_{1} W^{\prime} w\right)+\left(g(t)-c_{1}\right) w W \leq 0 \quad \text { on }[a, b] .
$$

Thus by the fact that $f(t)<b_{1}, g(t)>c_{1}$, and $w^{\prime}<0$, we have

$$
\begin{gathered}
\left(W w^{\prime}-W^{\prime} w\right)^{\prime}+b_{1}\left(w^{\prime} W-W^{\prime} w\right)<0 \quad \text { on }(a, b), \\
e^{b_{1} b}\left(W w^{\prime}-W^{\prime} w\right)(b)<e^{b_{1} a}\left(W w^{\prime}-W^{\prime} w\right)(a) .
\end{gathered}
$$

This is impossible and (ii) is proved. 
Proof of (iii). We use the same $v$ as in the proof of (ii), then $v \geq \lambda>1$ if $\bar{u} \geq \lambda u_{\alpha}$. Hence the proof of (ii) implies $v=0$ at some $t$.

Now we are ready to give the first global existence and large time behavior result.

Theorem 3.6. Suppose that $p>(n+l) /(n-2), \psi \not \equiv 0$ is a nonnegative radial continuous weak upper solution of (3.4) and the initial value $\phi \leq \psi$ in (3.1). Then (3.1) has a unique global solution $u$, which is $C_{b}$-mild when $-2<l<0$ and classical when $l \geq 0$, satisfying $0 \leq u(x, t) \leq \psi(x) \leq c(1+|x|)^{-(2+l) /(p-1)}$ on $R^{n} \times[0, \infty)$. Furthermore, if $(n+l) /(n-2)<p<p_{c}$ and $\psi$ is not an equilibrium of (3.1), then $\lim _{t \rightarrow+\infty}\|u(\cdot, t)\|_{L^{\infty}\left(R^{n}\right)}=0$. This is also true if $\phi \leq \lambda \psi$ for some constant $0<\lambda<1$ when $p \geq p_{c}$ (in this case, $\psi$ can be a equilibrium, and $0 \leq u \leq \lambda \psi)$.

Remark 1. When $(n+l) /(n-2)<p<(n+2+2 l) /(n-2)$, (3.1) has no nontrivial regular radial equilibrium (see Proposition 3.4). Also, by slightly extending Theorem 3.41 in [28], when $1<p \leq(n+l) /(n-2)$ the $\psi$ in the statement of Theorem 3.6 cannot exist.

Remark 2. From Theorem 3.6, it is easy to see that the regularity condition on $\bar{u}$ in (ii) of Proposition 3.5 can be reduced. This is also true for $\underline{u}$ as we shall see from Theorem 3.10.

Remark 3. As mentioned in $\S 0$, whenever $\|u(\cdot, t)\|_{\infty} \rightarrow 0$ as $t \rightarrow+\infty$ in the above theorem, we can actually say that $u(\cdot, t)$ decays in some weighted $L^{\infty}$ norms as $t \rightarrow+\infty$, because $0 \leq u(x, t) \leq \psi(x) \leq c(1+|x|)^{-(2+l) /(p-1)}$.

To prove Theorem 3.6, we need the following decay result on $\psi$ which is basically covered by Theorem 3.35 in [28].

Proposition 3.6' . Suppose $\psi$ is as stated in Theorem 3.6, then $\psi$ is nonincreasing in $r$ and $0<\psi(r) \leq((n+l)(2+l) /(p-1))^{1 /(p-1)} r^{-(2+l) /(p-1)}$.

Proof of Proposition 3.6' . Since $\psi$ is also a continuous weak superharmonic function, the mean-value inequality implies that $\psi>0$ (if we proceed by the maximum principle, $\psi(r)>c r^{2-n}$ at $\left.r=+\infty\right)$.

To prove the remaining part, let $j$ be the standard mollifier in $R^{n}$, and for each $\varepsilon>0$, let $j_{\varepsilon}(x)=\left(1 / \varepsilon^{n}\right) j(x / \varepsilon), \psi_{\varepsilon}=j_{\varepsilon} * \psi$, and $f_{\varepsilon}=j_{\varepsilon} *\left(|\cdot|^{l} \psi^{p}(\cdot)\right)$. Then $\Delta \psi_{\varepsilon}+f_{\varepsilon} \leq 0$ holds classically in $R^{n}$. Since $j$ is radial, by Lemma 1.4, $\psi_{\varepsilon}$ and $f_{\varepsilon}$ are also radial. Therefore

$$
\left(r^{n-1} \psi_{\varepsilon}^{\prime}(r)\right)^{\prime}+r^{n-1} f_{\varepsilon}(r) \leq 0 .
$$

Integrating from 0 to $r$ gives

$$
r^{n-1} \psi_{\varepsilon}^{\prime}(r)+\int_{0}^{r} s^{n-1} f_{\varepsilon}(s) d s \leq 0 .
$$

So $\psi_{\varepsilon}^{\prime}(r)<0(r>0)$ and

$$
\int_{0}^{r} \frac{\psi_{\varepsilon}^{\prime}(t)}{\psi_{\varepsilon}^{p}(t)} d t+\int_{0}^{r} d t \int_{0}^{t}\left(\frac{s}{t}\right)^{n-1} \frac{f_{\varepsilon}(s)}{\psi_{\varepsilon}^{p}(t)} d s \leq 0 .
$$

Hence $\psi_{\varepsilon}(r)$ is decreasing in $r$ and

$$
\frac{1}{p-1}\left(-\psi_{\varepsilon}^{1-p}(r)+\psi_{\varepsilon}^{1-p}(0)\right)+\int_{0}^{r} d t \int_{0}^{t}\left(\frac{s}{t}\right)^{n-1} \frac{f_{\varepsilon}(s)}{\psi_{\varepsilon}^{p}(t)} d s \leq 0 .
$$


Note $\psi_{\varepsilon} \rightarrow \psi$ pointwise and $f_{\varepsilon}(r) \rightarrow r^{l} \psi^{p}(r)$ as $\varepsilon \rightarrow 0^{+}$if $r \neq 0$. So by the Fatou Lemma

$$
\frac{1}{p-1}\left(-\psi^{1-p}(r)+\psi^{1-p}(0)\right)+\int_{0}^{r} d t \int_{0}^{t}\left(\frac{s}{t}\right)^{n-1} \frac{s^{l} \psi^{p}(s)}{\psi^{p}(t)} d s \leq 0
$$

and since $\psi$ is nonincreasing, we have

$$
\psi(r) \leq((n+l)(2+l) /(p-1))^{1 /(p-1)} r^{-(2+l) /(p-1)} .
$$

Proof of Theorem 3.6. The uniqueness of the global solution is a simple consequence of Theorem 2.3 (when $-2<l<0$ ) and Lemma 1.3 (when $l \geq 0$ ). To prove the remaining part, consider

$$
v_{t}=\Delta v+|x|^{l} v^{p},\left.\quad v\right|_{t=0}=\psi .
$$

Claim 1. (3.15) has a global nonnegative solution $v$, which is $C_{B}$-mild when $-2<l<0$ and classical when $l \geq 0$, satisfying that $v$ is radial in $x$ and $v$ is nonincreasing in $t \geq 0$. The proof of this claim is as follows. When $-2<l<0$, by Proposition 3.6', $\psi$ is bounded; by Lemma $2.2, \psi$ is a $C_{B}$-mild upper solution of (3.15). By Theorem 2.3, (3.15) has a nonnegative $C_{B}$-mild solution $v$ on $R^{n} \times\left[0, T_{\psi}\right)$ such that $v$ is radial in $x$. In virtue of Theorem 2.4, $T_{\psi}=+\infty$ and $v$ is nonincreasing in $t$. Claim 1 is proved when $-2<l<0$. When $l \geq 0$, it is routine to see that $\psi$ is also a c.w. upper solution of (3.15) (see Definition 1.1). Applying Lemma 1.2 with $\bar{u}=\psi$ and $\underline{u}=0$, we have that (3.15) has a classical global solution $v$ with $0 \leq v \leq \psi$. By Proposition 3.6 and Lemma 2.6, $v$ is radial in $x$ and $v$ is nonincreasing in $t$. Now the proof of Claim 1 is completed.

Since $\phi \leq \psi, v$ of (3.15) is an upper solution of (3.1). Applying Lemma 1.2 (when $l \geq 0$ ) and Theorem 2.4 (when $-2<l<0$ ) with $\bar{u}=v$ and $\underline{u}=0$, we have that (3.1) has a nonnegative global solution $u \leq v \leq \psi$. This together with Proposition 3.6' imply the global existence part of Theorem 3.6.

Now we turn to the large time behavior of $u$. By Claim 1, $v_{\infty}(x)=$ $\lim _{t \rightarrow+\infty} v(x, t)$ exists, $v_{\infty}$ is radial and $0 \leq v_{\infty} \leq \psi$.

Claim 2. The function $v_{\infty}$ is a (radial) regular solution of (3.4). Since even when $-2<l<0, v$ is also a c.w. solution of (3.15) (see the remark following Definition 2.1), we have for any $\tau>0$ and $\phi_{1} \in C_{0}^{\infty}\left(R^{n}\right)$

$$
\begin{aligned}
\int_{R^{n}} v & \left.(x, s+\tau) \phi_{1}(x) d x\right|_{\substack{s=0 \\
s=1}} ^{1} \\
& =\int_{0}^{1} d s \int_{R^{n}} v(x, s+\tau) \Delta \phi_{1}(x)+|x|^{l} v^{p}(x, s+\tau) \phi_{1}(x) d x .
\end{aligned}
$$

Let $\tau \rightarrow+\infty$, by the Lebesgue Dominated Convergence Theorem,

$$
0=\int_{R^{n}} v_{\infty} \Delta \phi_{1}+|x|^{l} v_{\infty}^{p} \phi_{1} d x
$$

Thus $v_{\infty}$ is a (bounded) distributional solution of (3.4). Now Claim 2 follows from the regularity theory for elliptic equations.

Claim 3. The function $v_{\infty}=0$ if $(n+l) /(n-2)<p<p_{c}$. We prove it in the following cases.

Case 1. $(n+l) /(n-2)<p<(n+2+2 l) /(n-2)$. By Proposition 3.4, (3.4) has no nontrivial regular radial solution. So $v_{\infty}=0$. 
Case 2. $(n+2+2 l) /(n-2)<p<p_{c}$. If $\psi$ is a regular upper solution of (3.4), by Claim 2, the fact $v_{\infty} \leq \psi$, and Proposition 3.5, either $v_{\infty} \equiv 0$ or $v_{\infty} \equiv \psi$. By assumption, $v_{\infty} \not \equiv \psi$ ( $\psi$ is not an equilibrium). So $v_{\infty} \equiv 0$. Now if $\psi$ is not regular, observe that since $v$ is nonincreasing in $t$, we can prove easily that for each $t>0, v(\cdot, t)$ is a c.w. upper solution of $(3.4)$. By regularity theory, $v(\cdot, t)$ is regular if $t>0$. Note also that $v(\cdot, t)$ is radial and $v_{\infty} \leq v(\cdot, t)$. Now by Proposition 3.5, either $v_{\infty} \equiv 0$ or $v_{\infty} \equiv v(\cdot, t)$ for all $t>0$ (here we should also use the fact that $v(\cdot, t)$ is nonincreasing in $t$ ). Since the latter implies $v_{\infty} \equiv \psi$ which contradicts our assumption, $v_{\infty} \equiv 0$.

Case 3. $p=(n+2+2 l) /(n-2)$. By the strong maximum principle for weakly superparabolic functions [8], it is easy to see that $v(x, t)<\psi(x)$ for $t>0$ and hence $v_{\infty}<\psi$. If $v_{\infty} \not \equiv 0$, by Claim 2 and Proposition 3.4, there exists $\beta>0$ such that

$$
v_{\infty}(r)=u_{\beta}^{E}(r)=\left(\frac{\beta \sqrt{(n+l)(n-2)}}{\beta^{2}+r^{2+l}}\right)^{(n-2) /(2+l)} .
$$

Let $\alpha$ be close to $\beta$ and $\alpha<\beta$ such that $\psi(0)>u_{\alpha}^{E}(0)\left(>u_{\beta}^{E}(0)\right)$. By a simple computation, we see that $u_{\alpha}^{E}$ and $u_{\beta}^{E}$ intersect at and only at $r_{\alpha, \beta}=$ $(\alpha \beta)^{1 /(2+l)}$ which goes to $\beta^{2 /(2+l)}$ as $\alpha \rightarrow \beta^{-}$. Obviously $u_{\alpha}^{E} \rightarrow u_{\beta}^{E}$ uniformly on $\left[0, \beta^{(2 /(l+2)}+1\right]$ as $\alpha \rightarrow \beta^{-}$. Thus there exists $\alpha<\beta$ such that $u_{\alpha}^{E}<\psi$ on $R^{n}$. When $-2<l<0$, by Lemma $2.2, u_{\alpha}^{E}$ is a $C_{B}$-mild lower solution of (3.15). In virtue of Theorem 2.4 (when $-2<l<0$ ) and Lemma 1.3 (when $l \geq 0)$ with $\bar{u}=v$ and $\underline{u}=u_{\alpha}^{E}$, we have $v \geq u_{\alpha}^{E}$ and hence $v_{\infty} \geq u_{\alpha}^{E}$. This is impossible because $u_{\alpha}^{E}(0)>u_{\beta}^{E}(0)=v_{\infty}(0)$. This completes the proof of Claim 3.

Now $\lim _{t \rightarrow+\infty} v(x, t)=0$. By the $L^{p}$ interior estimate and embedding, it is easy to see $\|v(\cdot, t)\|_{C^{\alpha}\left(\bar{B}_{R}\right)}<C_{R}<+\infty$ for $\alpha \in(0,2+l)$ and for all $t \geq 1$. Thus $v(x, t) \rightarrow 0$ locally uniformly on $R^{n}$ as $t \rightarrow+\infty$. But since $0 \leq v(x, t) \leq \psi(x) \leq C(1+|x|)^{-(2+l) /(p-1)}$ on $R^{n} \times[0, \infty)$, we have $\|v(\cdot, t)\|_{L^{\infty}\left(R^{n}\right)} \rightarrow 0$ as $t \rightarrow+\infty$. Now the fact $0 \leq u \leq v$ completes the proof of the large time behavior of $u$ when $(n+l) /(n-2)<p<p_{c}$.

To prove the large time behavior of $u$ when $p \geq p_{c}$ and $\phi \leq \lambda \psi$ for some $0<\lambda<1$, we follow the same line of reasoning. First replace $\psi$ in (3.15) by $\lambda \psi$ and denote the corresponding solution of (3.15) by $v^{\lambda}$. Since $\lambda \psi$ is also a c.w. upper solution of (3.4), Claim 1 is true for $v^{\lambda}$. Claim 2 holds for $v_{\infty}^{\lambda}=\lim _{t \rightarrow+\infty} v^{\lambda}$ by the same argument there. To prove $v_{\infty}^{\lambda} \equiv 0$, noticing $v_{\infty}^{\lambda} \leq v_{\lambda} \leq \lambda \psi$, we have $v_{\infty}^{\lambda} / \lambda \leq \psi$. Now consider the global solution $v$ of (3.15) (keep $\left.\left.v\right|_{t=0}=\psi\right)$. Since $v_{\infty}^{\lambda} / \lambda$ is a c.w. lower solution of $(3.15)(1 / \lambda>1$ and $v_{\infty}^{\lambda}$ is an equilibrium), we have by the comparison principle (see Theorem 2.4 when $-2<l<0$ and Lemma 1.3 when $l \geq 0$ ) that $v_{\infty}^{\lambda} / \lambda \leq v$ and hence $v_{\infty}^{\lambda} / \lambda \leq v_{\infty}=\lim _{t \rightarrow+\infty} v(\cdot, t)$ (note Claim 1 and Claim 2 are of course true for $v$ ). If $v_{\infty}^{\lambda} \not \equiv 0$, then $v_{\infty}^{\lambda}$ and $v_{\infty}$, as nontrivial regular solutions of (3.4), satisfy $\lim _{r \rightarrow+\infty} v_{\infty}^{\lambda} / v_{\infty}=1$ by Proposition 3.4, a contradiction! Therefore $v_{\infty}^{\lambda} \equiv 0$. Similarly as for the case $(n+l) /(n-2)<p<p_{c}$, we have $\|u(\cdot, t)\|_{L^{\infty}\left(R^{n}\right)} \leq$ $\left\|v^{\lambda}(\cdot, t)\right\|_{L^{\infty}\left(R^{n}\right)} \rightarrow 0$ as $t \rightarrow+\infty$.

We shall use Theorem 3.6 to obtain more detailed global existence and large 
time behavior result. To this end, we use the solutions of $(3.4)$ and $(3.5)_{R}$ to construct various upper and lower solutions for (3.1). The following observation will be useful (for notation used in the following statement, see Proposition 3.4).

Proposition 3.7. (i) When $(n+l) /(n-2)<p<(n+2+2 l) /(n-2)$, the graph of $u_{R}^{D}(r)$ intersects (transversely) that of $u_{s}(r)$ for every $R>0$.

(ii) When $p=(n+2+2 l) /(n-2)$, the graph of $u_{\lambda}^{E}(r)$ intersects (transversely) that of $u_{s}(r)$ at and only at

$$
r=\left(\lambda\left(\sqrt{\frac{n+l}{n-2}} \pm \sqrt{\frac{2+l}{n-2}}\right)\right)^{2 /(2+l)} .
$$

(iii) When $(n+2+2 l) /(n-2)<p<p_{c}$, the graph of $u_{\alpha}(r)$ oscillates around that of $u_{s}(r)$ for every $\alpha>0$.

(iv) When $p \geq p_{c}$, the graph of $u_{\alpha}$ does not intersect that of $u_{s}$ (i.e., $u_{\alpha}(r)<$ $u_{s}(r)$ for all $\left.r \geq 0\right)$ for every $\alpha>0$. Furthermore, $u_{\alpha}(r)$ is increasing with respect to $\alpha>0$.

Remark 1 . The above result can be observed from the phase diagram in [20] when $l=0$.

Remark 2. The following proof of the first part of (iv) is inspired by an idea in [36].

Proof of Proposition 3.7. To prove (i), let $v(t)=r^{(2+l) /(p-1)} u_{R}^{D}(r), t=\log r$. Then

$$
v^{\prime \prime}+c_{0} v^{\prime}+v\left(v^{p-1}-L^{p-1}\right)=0 \quad \text { on }(-\infty, \log R]
$$

where

$$
c_{0}=\frac{n-2}{p-1}\left(p-\frac{n+2+2 l}{n-2}\right)
$$

Observe that

$$
v(\log R)=0 \quad \text { and } \quad v>0, \quad v(t) \rightarrow 0 \quad \text { as } t \rightarrow-\infty .
$$

So $v$ must achieve its maximum value at some $t_{0}$. By (3.16),

$$
v\left(t_{0}\right)\left(v^{p-1}\left(t_{0}\right)-L^{p-1}\right) \geq 0,
$$

hence $v\left(t_{0}\right) \geq L$. By the uniqueness for O.D.E., $v\left(t_{0}\right)>L$. The proof of (i) is completed. (ii) follows from direct calculation. (iii) follows from (iv) of Proposition 3.4 and (i) of Proposition 3.5.

Now we prove (iv). Let $v(t)=r^{(2+l) /(p-1)} u_{\alpha}(r), t=\log r$. Then (3.16) is true on $(-\infty,+\infty)$ with $v>0$ and $\lim _{t \rightarrow-\infty} v(t)=0 ; \lim _{t \rightarrow+\infty} v(t)=L$. If the first conclusion of (iv) is not true, let $t_{0}=\min \{t \mid v(t)=L\}$. As in the proof of Proposition 3.5, $v^{\prime}>0$ on $\left[-\infty, t_{0}\right]$ and $v^{\prime}(t) \rightarrow 0$ as $t \rightarrow-\infty$. Let $q(v)=v^{\prime}(t)$. Then

$$
\frac{d q}{d v}+c_{0}+\frac{v\left(v^{p-1}-L^{p-1}\right)}{q}=0 \quad \text { on }(0, L],
$$

$q>0$ on $(0, L]$ and $q(v) \rightarrow 0$ as $v \rightarrow 0^{+}$. Therefore in the $q-v$-plane, the graph of $q=q(v)$ intersects all lines $q=\mu(L-v)$ with $\mu>0$. For each 
$\mu>0$, denote the intersection with the smallest $v$ coordinate by $\left(v_{\mu}, q\left(v_{\mu}\right)\right)$. Then $(d q / d v)\left(v_{\mu}\right) \geq-\mu$ and

$$
\begin{aligned}
\frac{d q}{d v}\left(v_{\mu}\right) & =-c_{0}-\frac{v_{\mu}^{p}-L^{p-1} v_{\mu}}{\mu\left(L-v_{\mu}\right)} \\
& =-c_{0}+\frac{p \bar{v}_{\mu}^{p-1}-L^{p-1}}{\mu} \text { for some } \bar{v}_{\mu} \in\left(v_{\mu}, L\right) \\
& <-c_{0}+\frac{(p-1) L^{p-1}}{\mu} .
\end{aligned}
$$

Thus

$$
\begin{gathered}
-\mu<-c_{0}+(p-1) L^{p-1} / \mu, \\
0<\mu^{2}-c_{0} \mu+(p-1) L^{p-1} \text { for all } \mu>0 .
\end{gathered}
$$

Then $c_{0}^{2}-4(p-1) L^{p-1}<0$. But when $p \geq p_{c}$, by a long but straightforward calculation, $c_{0}^{2}-4(p-1) L^{p-1} \geq 0$. We reach a contradiction. The first part of (iv) is proved. To prove the second part, we note from the first part, $0<$ $v<L$ on $(-\infty,+\infty)$ and hence $v^{\prime}(t)>0$ on $(-\infty,+\infty)$ (this can be seen from (3.16) and a similar argument in the proof of (ii) of Proposition 3.5). Since $v^{\prime}(t)=r d\left(r^{(2+l) /(p-1)} u_{\alpha}(r)\right) / d r, d\left(r^{(2+l) /(p-1)} u_{\alpha}(r)\right) / d r>0$ if $r \neq 0$. By this and the fact $u_{\alpha}(r)=\alpha^{(2+l) /(p-1)} u_{1}(\alpha r)$ ((iv) of Proposition 3.4), one has $\partial u_{\alpha}(r) / \partial \alpha>0$ if $r>0$.

Next, we use the solutions of (3.4) and $(3.5)_{R}$ to construct some "good" upper and lower solutions of (3.1). Before doing so, we introduce some notation. When $(n+l) /(n-2)<p<(n+2+2 l) /(n-2)$, let

$$
\begin{aligned}
& r_{m}^{D}(R)=\min \left\{r \in[0, R] \mid u_{R}^{D}(r)=u_{s}(r)\right\}, \\
& r_{M}^{D}(R)=\max \left\{r \in[0, R] \mid u_{R}^{D}(r)=u_{s}(r)\right\} .
\end{aligned}
$$

By Proposition 3.7, they are well defined. Furthermore, from (ii) of Proposition 3.4, we have

$$
r_{m}^{D}(R)=R r_{m}^{D}(1), \quad r_{M}^{D}(R)=R r_{M}^{D}(1) .
$$

When $p=(n+2+2 l) /(n-2)$, denote by $r_{m}^{E}(\lambda)$ and $r_{M}^{E}(\lambda)$ the $r$ coordinates of the two intersections of the graphs of $u_{\lambda}^{E}(r)$ and $u_{s}(r)$ with $r_{m}^{E}(\lambda)<r_{M}^{E}(\lambda)$ (see (ii) of Proposition 3.7). We observe

$$
r_{m}^{E}(\lambda)=\lambda^{2 /(2+l)} r_{m}^{E}(1), \quad r_{M}^{E}(\lambda)=\lambda^{2 /(2+l)} r_{M}^{E}(1) .
$$

When $(n+2+2 l) /(n-2)<p<p_{c}$, let $r_{1}(\alpha)=\min \left\{r \geq 0 \mid u_{\alpha}(r)=u_{s}(r)\right\}$, $r_{2}(\alpha)=\min \left\{r>r_{1}(\alpha) \mid u_{\alpha}(r)=u_{s}(r)\right\}$. They are well defined by (iii) of Proposition 3.7. From (iv) of Proposition 3.4, we have

$$
r_{i}(\alpha)=r_{i}(1) / \alpha, \quad i=1,2 .
$$

Proposition 3.8. (i) When $(n+l) /(n-2)<p<(n+2+2 l) /(n-2)$, define in $R^{n}$

$$
\begin{aligned}
& \bar{u}_{R}(x)= \begin{cases}u_{s}(|x|), & |x|>r_{m}^{D}(R), \\
u_{R}^{D}(|x|), & |x| \leq r_{m}^{D}(R),\end{cases} \\
& \underline{u}_{R}(x)= \begin{cases}u_{s}(|x|), & |x|>r_{M}^{D}(R), \\
u_{R}^{D}(|x|), & |x| \leq r_{M}^{D}(R) .\end{cases}
\end{aligned}
$$


Then for every $R>0, \bar{u}_{R}\left(\underline{u}_{R}\right)$ is a c.w. upper (lower) solution of (3.4).

(ii) When $p=(n+2+2 l) /(n-2)$, define in $R^{n}$

$$
\begin{aligned}
& \bar{u}_{\lambda}^{E}(x)= \begin{cases}u_{s}(|x|), & |x|>r_{m}(\lambda), \\
u_{\lambda}^{E}(|x|), & |x| \leq r_{m}(\lambda),\end{cases} \\
& \underline{u}_{\lambda}^{E}(x)= \begin{cases}u_{s}(|x|), & |x|>r_{M}(\lambda), \\
u_{\lambda}^{E}(|x|), & |x| \leq r_{M}(\lambda) .\end{cases}
\end{aligned}
$$

Then for every $\lambda>0, \bar{u}_{\lambda}^{E}\left(\underline{u}_{\lambda}^{E}\right)$ is a c.w. upper (lower) solution of (3.4).

(iii) When $p \geq(n+2+2 l) /(n-2)$, for every $\lambda>0, \alpha>0$, and $0<\beta \leq 1$, $\beta u_{\alpha}$ and $\beta u_{\lambda}^{E}$ are c.w. upper solutions of (3.4) and for $\beta>1$, they are c.w. lower solutions of (3.4).

(iv) When $(n+2+2 l) /(n-2)<p<p_{c}$, define in $R^{n}$

$$
\begin{aligned}
& \tilde{u}_{\alpha}= \begin{cases}u_{s}(|x|), & |x|>r_{1}(\alpha), \\
u_{\alpha}(|x|), & |x| \leq r_{1}(\alpha),\end{cases} \\
& \hat{u}_{\alpha}= \begin{cases}u_{s}(|x|), & |x|>r_{2}(\alpha), \\
u_{\alpha}(|x|), & |x| \leq r_{2}(\alpha) .\end{cases}
\end{aligned}
$$

Then $\tilde{u}_{\alpha}\left(\hat{u}_{\alpha}\right)$ is a c.w. upper (lower) solution of (3.4).

Proof. We just prove (i); the proofs of (ii)-(iv) are either trivial or similar to (i). For all $\phi_{1} \in C_{0}^{\infty}\left(R^{n}\right)$ with $\phi_{1} \geq 0$, we need to show

$$
\int_{R^{n}} \bar{u}_{R} \Delta \phi_{1}+|x|^{l}\left(\bar{u}_{R}\right)^{p} \phi_{1} \leq 0
$$

Let $j$ be the standard mollifier in $R^{n}$, and for $\varepsilon>0$, let $j_{\varepsilon}(x)=j(x / \varepsilon) / \varepsilon^{n}$, $f(x)=|x|^{l} u_{R}^{D}$. Then

$$
\Delta\left(j_{\varepsilon} * u_{R}^{D}\right)+j_{\varepsilon} * f=0
$$

classically in $B_{R-\varepsilon}$. Denote $\left\{|x| \leq r_{m}^{D}(R)\right\}$ by $B$; then for small $\varepsilon>0$,

$$
\begin{aligned}
\int_{B}\left(j_{\varepsilon} * u_{R}^{D}\right) \Delta \phi_{1}+\left(j_{\varepsilon} * f\right) \phi_{1}= & \int_{B} \Delta\left(j_{\varepsilon} * u_{R}^{D}\right) \phi_{1}+\left(j_{\varepsilon} * f\right) \phi_{1} \\
& +\int_{\partial B} \frac{\partial \phi_{1}}{\partial \eta}\left(j_{\varepsilon} * u_{R}^{D}\right)-\phi_{1} \frac{\partial\left(j_{\varepsilon} * u_{R}^{D}\right)}{\partial \eta} \\
= & \int_{\partial B} \frac{\partial \phi_{1}}{\partial \eta}\left(j_{\varepsilon} * u_{R}^{D}\right)-\phi_{1} \frac{\partial\left(j_{\varepsilon} * u_{R}^{D}\right)}{\partial \eta}
\end{aligned}
$$

( $\eta$ is the outer normal of $\partial B$ ). Let $\varepsilon \rightarrow 0^{+}$, then

$$
\int_{B} u_{R}^{D} \Delta \phi_{1}+f \phi_{1}=\int_{\partial B} \frac{\partial \phi_{1}}{\partial \eta} u_{R}^{D}-\phi_{1} \frac{\partial u_{R}^{D}}{\partial \eta}
$$

$\left(u_{R}^{D}\right.$ is classical at $\left.x \neq 0\right)$. It is easy to see

This and (3.21) yield

$$
\int_{B^{c}} u_{s} \Delta \phi_{1}+|x|^{l} u_{s}^{p} \phi_{1}=\int_{\partial B}-u_{s} \frac{\partial \phi_{1}}{\partial \eta}+\frac{\partial u_{s}}{\partial \eta} \phi_{1} .
$$

$$
\int_{R^{n}} \bar{u}_{R} \Delta \phi_{1}+|x|^{l}\left(\bar{u}_{R}\right)^{p} \phi_{1}=\int_{\partial B}\left(\frac{\partial u_{s}}{\partial \eta}-\frac{\partial u_{R}^{D}}{\partial \eta}\right) \phi_{1} .
$$

Since $u_{s}^{\prime}\left(r_{m}^{D}(R)\right)<\left(u_{R}^{D}\right)^{\prime}\left(r_{m}^{D}(R)\right)$, the proof is finished.

We are ready to give a global existence and large time behavior result more specific than that of Theorem 3.6. 
Theorem 3.9. (i) When $(n+l) /(n-2)<p<p_{c}$, if the initial value $\phi \leq u_{s}$ on $R^{n}$, then (3.1) has a (unique) global solution $u$ which is $C_{B}$-mild when $-2<$ $l<0$ and classical when $l \geq 0$, satisfying $0 \leq u \leq u_{s}$ and $\|u(\cdot, t)\|_{L^{\infty}\left(R^{n}\right)} \rightarrow 0$ as $t \rightarrow+\infty$.

(ii) When $p \geq p_{c}$, if $\phi \leq \beta u_{s}$ for some constant $0<\beta<1$, then the conclusion of (i) still holds.

(iii) In (i) and (ii), if $\phi \leq \beta u_{s}$, for some constant $0<\beta<1$, then $u \leq \beta u_{s}$.

(iv) When $p \geq(n+2+2 l) /(n-2)$, if $\phi \leq \beta u_{\alpha}$ (or $\left.\phi \leq \beta u_{\lambda}^{E}\right)$ for some $0<$ $\beta<1$ and some equilibrium $u_{\alpha}\left(u_{\lambda}^{E}\right)$ of (3.1) mentioned in Proposition 3.4 , then the conclusion of (i) is true with " $u \leq u_{s}$ " replaced by " $u \leq \beta u_{\alpha}$ (or $\beta u_{\lambda}^{E}$ )". Furthermore, if $\beta=1$, then (3.1) has a (unique) global solution $u \leq u_{\alpha}\left(\right.$ or $\left.u_{\lambda}^{E}\right)$.

Remark. As we mentioned in $\S 0$, if we replace in (ii) the condition " $\phi \leq \beta u_{s}$ " by " $\phi \leq u_{s}$ ", then the conclusion of (ii) is not true because by (iv) of Proposition 3.7, all radial regular equilibrium of (3.1) stay below $u_{s}$. However, let $\beta_{k} \uparrow 1$ and if we take the initial value in (3.1) as $\beta_{k} \phi$, then by (ii), for each $k,(3.1)$ has a bounded global solution $u_{k} \leq u_{s}$. By the comparison principle (see Theorem 2.4 when $-2<l<0$ and Lemma 1.3 when $l \geq 0), u_{k} \leq u_{k+1}$ on $R^{n} \times[0,+\infty)$ and $u_{k} \leq u$ on $R^{n} \times\left[0, T_{\phi}\right.$ ) (note at least the local solution $u$ of (3.1) exists by Theorem 2.3 and Theorem 2.5). By the continuity of the solution of (3.1) with respect to the initial value (this can be seen from the Gronwall inequality and the proofs of Theorem 2.3 and Theorem 2.5), $u_{k} \rightarrow u$ (at least) pointwise on $R^{n} \times\left[0, T_{\phi}\right)$ as $k \rightarrow+\infty$. On the other hand, on $R^{n} \times[0,+\infty)$, $\tilde{u}=\lim _{k \rightarrow+\infty} u_{k}$ exists and $\tilde{u}=u$ if $0<t \leq T_{\phi}, 0 \leq \tilde{u} \leq u_{s}$. Also, it is easy to see that $\tilde{u}$ is at least a global distributional solution of (3.1). Therefore in (ii), if we only have $\phi \leq u_{s},(3.1)$ at least has a global distributional solution $\tilde{u} \leq u_{s}$ which is, before time $T_{\phi}, C_{B}$-mild when $-2<l<0$ or classical when $l \geq 0$. Note in any case, $\tilde{u}$ is classical outside $\{0\} \times[0,+\infty)$ by the regularity theory for parabolic equations. The question of large time behavior of $\tilde{u}$ remains open.

Proof of Theorem 3.9. We first prove (i). We shall use Proposition 3.8 to find a positive radial c.w. upper solution of (3.4) which is above $\phi$ and below $u_{s}$, then (i) follows from Theorem 3.6. To this end, observe that since $\phi$ is bounded and $\phi \leq u_{s}$, there exists $R_{1}>0$ such that $\phi \leq u_{s}\left(R_{1}\right)$.

Case 1. $(n+l) /(n-2)<p<(n+2+2 l) /(n-2)$. By (3.18), there exists an $R>0$ such that $r_{m}^{D}(R)=R_{1}$. Since $u_{R}^{D}(r)$ is decreasing on $[0, R]$ (see Proposition 3.4), $\phi(x) \leq u_{R}^{D}(x)$ if $|x| \leq R_{1}=r_{m}^{D}(R)$. Thus $\phi \leq \bar{u}_{R}\left(\leq u_{s}\right)$ on $R^{n} \quad\left(\bar{u}_{R}\right.$ is defined in Proposition 3.8). This $\bar{u}_{R}$ is what we seek.

Case 2. $p=(n+2+2 l) /(n-2)$. Using (3.19), we can find a $\lambda>0$ such that $\phi \leq \bar{u}_{\lambda}^{E}\left(\leq u_{s}\right)$ and by Proposition 3.8, $\bar{u}_{\lambda}^{E}$ is what we want.

Case 3. $(n+2+2 l) /(n-2)<p<p_{c}$. This time we use (3.20) and Proposition 3.8 to find a $\tilde{u}_{\alpha}$ desired.

The proof of (i) is now complete.

To prove (ii), we look for a constant $0<\beta_{2}<1$ and a radial equilibrium $u_{\alpha_{1}}$ mentioned in Proposition 3.4 such that $\phi \leq \beta_{2} u_{\alpha_{1}}$ on $R^{n}$, then (ii) follows from (iv) which is immediate from Theorem 3.6 (note if $0<\beta<1, \beta u_{\alpha}$ is a c.w. upper solution of (3.4)). Since $\phi \leq \beta u_{s}, \lim \sup _{|x| \rightarrow+\infty}|x|^{(2+l) /(p-1)} \phi(x)<L$. 
By (iv) of Proposition 3.4, $L=\lim _{r \rightarrow+\infty} r^{(2+l) /(p-1)} u_{1}(r)$. Therefore, there exist $1>\beta_{0}>0$ and $R>1$ such that

$$
\phi(x) \leq \beta_{0} u_{1}(x) \text { for }|x| \geq R .
$$

Obviously, there exists $1>\delta>0$ such that $\phi(x) \leq \beta_{0} u_{s}(\delta)$. From (iv) of Proposition 3.4 again,

$$
\begin{aligned}
u_{\alpha}(r) & =\alpha^{(2+l) /(p-1)} u_{1}(\alpha r) \\
& =\left((\alpha r)^{(2+l) /(p-1)} u_{1}(\alpha r)\right) r^{-(2+l) /(p-1)} \rightarrow L r^{-(2+l) /(p-1)}=u_{s}(r)
\end{aligned}
$$

as $\alpha \rightarrow \infty$. So there exist $1>\beta_{1}>\beta_{0}$ and $\alpha_{0}>1$ such that $\beta_{0} u_{s}(\delta)<$ $\beta_{1} u_{\alpha_{0}}(\delta)$. Thus

$$
\phi(x)<\beta_{1} u_{\alpha_{0}}(\delta) \leq \beta_{1} u_{\alpha_{0}}(|x|) \quad \text { if }|x| \leq \delta
$$

Since $u_{\alpha} \rightarrow u_{s}$ uniformly on $[\delta, R]$ as $\alpha \rightarrow+\infty$ and $\phi<u_{s}$, there exists $1>\beta_{2}>\beta_{1}$ and $\alpha_{1}>\alpha_{0}$ such that

$$
\phi(x)<\beta_{2} u_{\alpha_{1}}(|x|) \quad \text { if } \delta \leq|x| \leq R .
$$

Combining (3.22)-(3.24) and the fact that $u_{\alpha}$ is increasing in $\alpha$ (see (iv) of Proposition 3.7), we have $\phi \leq \beta_{2} u_{\alpha_{1}}<\beta_{2} u_{s}$ on $R^{n}$. We finish the proof of (ii).

To prove (iii), first we notice that when $(n+l) /(n-2)<p<p_{c}$ if we replace $\phi$ in the proof of (i) by $\phi / \beta$ ( $\leq u_{s}$ by the assumption), then we can find a radial c.w. upper solution $\psi$ of (3.4) such that $\phi / \beta \leq \psi \leq u_{s}$, i.e., $\phi \leq \beta \psi \leq \beta u_{s}$. Since $\beta \psi$ is also a c.w. upper solution of (3.4), by Theorem 3.6, $u \leq \beta \psi$ (here we should notice that the global solution of (3.1) satisfying the properties in (i) is unique by Theorem 2.3 when $-2<l<0$ and Lemma 1.3 when $l \geq 0$ ) and hence $u \leq \beta u_{s}$. Next, when $p \geq p_{c}$, by examining the proof of (ii) closely, $\beta_{0}$ can be chosen arbitrarily close to $\beta, \beta_{1}$ and $\beta_{2}$ can be chosen arbitrarily close to $\beta_{0}$ and $\beta_{1}$ respectively. Hence $\beta_{2}$ can be arbitrarily close to $\beta$. Since $\phi \leq \beta_{2} u_{\alpha_{1}}$ and $\beta_{2} u_{\alpha_{1}}$ is a c.w. upper solution of (3.4), we have $u \leq \beta_{2} u_{\alpha_{1}}\left(\leq \beta_{2} u_{s}\right)$. Letting $\beta_{2} \rightarrow \beta$, we have $u \leq \beta u_{s}$. (iii) is now proved.

Next, we turn to the finite time blow-up results. The following theorem is in a direction opposite to that of Theorem 3.6.

Theorem 3.10. Suppose that $\psi \not \equiv 0$ is a radial nonnegative c.w. lower solution of (3.4) which is not a solution of (3.4) when $(n+2+2 l) /(n-2) \leq p<p_{c}$.

(i) When $1<p<p_{c}$, if the initial value $\phi$ in $(3.1) \geq \psi, \phi$ is bounded when $-2<l<0$, and $\phi(x) \leq c|x|^{-l /(p-1)}$ at $|x|=+\infty$ when $l \geq$ 0 , then the local solution of (3.1), whose existence and uniqueness are assured by Theorem 2.3 (when $l<0$ ) and Theorem 2.5 (when $l \geq 0$ ), satisfies that $T_{\phi}<+\infty$ and hence $\lim _{t \rightarrow T_{\phi}^{-}}\|u(\cdot, t)\|_{L^{\infty}\left(R^{n}\right)}=+\infty$ when $-2<l<0$, and $\lim _{t \rightarrow T_{\phi}^{-}}\|u(\cdot, t) w(\cdot)\|_{L^{\infty}\left(R^{n}\right)}=+\infty$ when $l \geq 0$, where $w(x)=(1+|x|)^{l /(p-1)}$.

(ii) When $p \geq p_{c}$, if the conditions on $\phi$ in (i) hold with " $\phi \geq \psi$ " replaced by " $\phi \geq \beta \psi$ " for some constant $\beta>1$, then the conclusion of (i) is still true. 
Remark 1. When $l \geq 0$, we can prove that if $\phi \geq \psi$ when $1<p<p_{c}$, or if $\phi \geq \beta \psi(\beta>1)$ when $p \geq p_{c}$, then (3.1) has no global classical solution $u$ with $0 \leq u(x, t) \leq C(T)(1+|x|)^{(2-l) /(p-1)}$ on $R^{n} \times[0, T]$ for any $T>0$. (This result is slightly different from Theorem 3.10.) The proof of this is the same as that of Theorem 3.10.

Remark 2. When $l=0$ and $1<p \leq(n+2) / n$, Theorem 3.10 is immediate from the Fujita-type blow-up results in $[11,22]$. This is also true when $l>-2$ and $1<p<(n+2+l) / n$ by the blow-up result in [4].

Proof of Theorem 3.10. To prove (i), suppose contrary to the conclusion, that $T_{\phi}=+\infty$. Then $u$ is a $\left(C_{B}\right.$-mild when $l<0$, classical when $\left.l \geq 0\right)$ upper solution of

$$
v_{t}=\Delta v+|x|^{l} v^{p},\left.\quad v\right|_{t=0}=\psi .
$$

Applying Theorem 2.4 (when $-2<l<0$ ) and Lemma 1.2 (when $l \geq 0$ ) with $\bar{u}=u$ and $\underline{u}=0$, we have that (3.25) has a global solution $v$, which is $C_{B}$-mild when $-2<l<0$ and classical when $l \geq 0$, satisfying $0 \leq v \leq u$. Furthermore, $v$ is radial in $x$ and $v$ is nondecreasing in $t$, by Theorem 2.3, Theorem 2.4, and Lemma 2.6 (note by Theorem 2.5 when $l \geq 0,\|u(\cdot, t) w(\cdot)\|_{L^{\infty}\left(R^{n}\right)}$ is locally bounded on $[0,+\infty)$ and hence $v$ satisfies the condition in Lemma 2.6). Now, let $v_{\infty}(x)=\lim _{t \rightarrow+\infty} v(x, t)$, then $v_{\infty}$ is radial and $\psi \leq v \leq v_{\infty}$. By Corollary 3.2, $v_{\infty} \in L_{\text {loc }}^{1}\left(R^{n} \backslash\{0\}\right)$.

Claim. The function $v_{\infty}$ is a (radial) solution (either regular or singular at $x=0)$ of (3.4). To prove this, let $\tau>0$ and $\phi_{1} \in C_{0}^{\infty}\left(R^{n} \backslash\{0\}\right)$, then

$$
\begin{aligned}
\int_{R^{n}} v & \left.(x, s+\tau) \phi_{1}(x) d x\right|_{\mid s=0} ^{s=1} \\
& =\int_{0}^{1} d s \int_{R_{n}} v(x, s+\tau) \Delta \phi_{1}(x)+|x|^{l} v^{p}(x, s+\tau) \phi_{1}(x) d x .
\end{aligned}
$$

Taking nonnegative $\phi_{1}$, from the fact $v_{\infty} \in L_{\text {loc }}^{1}\left(R^{n} \backslash\{0\}\right)$ and Fatou's Lemma, one sees that $|\cdot|^{l} v_{\infty}^{p} \in L_{\text {loc }}^{1}\left(R^{n} \backslash\{0\}\right)$. Letting $\tau \rightarrow+\infty$, by the Lebesgue Dominated Convergence Theorem,

$$
0=\int_{R^{n}} v_{\infty} \Delta \phi_{1}+|\cdot|^{l} v_{\infty}^{p} \phi_{1} d x .
$$

Taking radial $\phi_{1}$, it is easy to see that

$$
\left(r^{n-1} v_{\infty}^{\prime}(r)\right)^{\prime}+r^{n+l-1} v_{\infty}^{p}(r)=0 \quad \text { on }(0,+\infty)
$$

in the distributional sense. Since $|\cdot|^{l} v_{\infty}^{p} \in L_{\text {loc }}^{1}\left(R^{n} \backslash\{0\}\right)$, we have $r^{n+l-1} v_{\infty}^{p}(r) \in$ $L_{\text {loc }}^{1}(0,+\infty)$ and therefore by a bootstrap argument, $v_{\infty}(r) \in C^{2}(0,+\infty)$. Thus $v_{\infty}$ is either a regular or a singular (at $|x|=0$ ) solution of (3.4). The proof of the claim is completed.

Next, we prove by using elliptic theory that $v_{\infty}$ cannot be a nontrivial solution of (3.4), and then (i) follows.

Case 1. $1<p \leq(n+l) /(n-2)$. By Theorem 3.41 in [28], (3.4) has no nontrivial solution even on exterior domains. So $v_{\infty}$ cannot be a solution of (3.4).

Case 2. $(n+l) /(n-2)<p<(n+2+2 l) /(n-2)$. In this case, (3.4) has no nontrivial radial regular solution (see (ii) of Proposition 3.4). On the 
other hand, $v_{\infty} \not \equiv u_{s}$, for otherwise, $\phi \leq v_{\infty} \equiv u_{s}$ and hence by Theorem 3.9, $v_{\infty} \equiv 0$. A contradiction. If $v_{\infty}$ is one of the other singular solutions of (3.4), say, $v_{\infty}=U_{s}$ (mentioned in Proposition 3.4), we shall also reach a contradiction as follows. By (i) of Proposition 3.5, there exist $r_{k} \rightarrow 0^{+}$such that $U_{s}\left(r_{k}\right)=u_{s}\left(r_{k}\right)$. This and (3.18) imply that there exist $R_{k} \rightarrow 0^{+}$such that $u_{R_{k}}^{D}(r)$ and $U_{s}(r)$ intersect at $r=\bar{R}_{k}$ with $U_{s}(r) \geq u_{R_{k}}^{D}(r)$ when $r \leq \bar{R}_{k}$. (So $\bar{R}_{k}$ is the $r$-coordinate of the first intersection of $u_{R_{k}}^{D}$ and $U_{s}$.) Define

$$
\bar{U}(r)= \begin{cases}u_{R_{k}}^{D}(r), & \text { if } r \leq R_{k}, \\ U_{s}(r), & \text { if } r>R_{k} .\end{cases}
$$

In the following, we assume $R_{k}$ is chosen so that $\psi \leq \bar{U}$ on $R^{n}$. By the proof of Proposition 3.8, $\bar{U}$ is a c.w. upper solution of (3.4) and hence of (3.25). By the comparison principle (see Theorem 2.4 when $-2<l<0$ and Lemma 1.3 when $l \geq 0), v \leq \bar{U}$ on $R^{n}$. Hence $v_{\infty} \leq \bar{U}\left(\leq U_{s}=v_{\infty}\right)$. This is impossible.

Case 3. $p=(n+2+2 l) /(n-2)$. As in Case 2, $v_{\infty} \not \equiv u_{s}$. Suppose $v_{\infty}$ is another radial singular solution of (3.4), then the fact that $v_{\infty}$ oscillates around $u_{s}$ near $r=0$ (see (iii) of Proposition 3.4), (3.19) and the same reasoning as in Case 2 lead us to a contradiction. Also, $v_{\infty}$ cannot be a radial regular solution of (3.4). This can be proved by finding a regular solution $u_{\alpha}^{E}$ of (3.4) so that $\psi \leq u_{\alpha}^{E}$ and $u_{\alpha}^{E}(0)<v_{\infty}(0)$, if $v_{\infty}$ is indeed a radial regular solution of (3.4). The construction of $u_{\alpha}^{E}$ is similar to that of $u_{\alpha}^{E}$ in Case 3 of Claim 3 in the proof of Theorem 3.6. We omit the details.

Case 4. $(n+2+2 l) /(n-2)<p<p_{c}$. First, as in Case 2, $v_{\infty}$ cannot be $u_{s}$. Next, if $v_{\infty}$ is a (radial) regular solution of (3.4), we still have a contradiction as follows. Since $v$ is nondecreasing in $t$, it is easy to see for each $t>0$, $v(\cdot, t)$ is a (radial) regular lower solution of $(3.4)$ with $v(\cdot, t) \leq v_{\infty}$. By (ii) of Proposition 3.5, $v(\cdot, t) \equiv v_{\infty}$ for each $t>0$ and hence $\psi=v(\cdot, 0)=v_{\infty}$. This contradicts the assumption that $\psi$ is not a solution of (3.4).

Now the proof of (i) is completed.

To prove (ii), replace the initial value $\psi$ in (3.25) by $\beta \psi$. If the conclusion of (ii) is untrue, then as in the proof of (i), (3.25) has a global solution $v$ such that $v$ is radial in $x$ and nondecreasing in $t$ (note $\beta \psi$ with $\beta>1$ is a c.w. lower solution of (3.4)), and $v_{\infty}(x)=\lim _{t \rightarrow+\infty} v(x, t)$ is a radial solution (regular or singular at $x=0$ ) of (3.4). If $v_{\infty}$ is singular, then Proposition 3.4 implies $v_{\infty} \equiv u_{s}$ and hence $\beta \psi \leq u_{s}, \psi \leq u_{s} / \beta$. By (ii) of Theorem 3.9, the solution $v_{\psi}$ of (3.25) (keep $\left.v\right|_{t=0}=\psi$ ) decays to zero as $t \rightarrow+\infty$ if $\psi \leq u_{s} / \beta$. But $v_{\psi} \geq \psi \not \equiv 0$, so we reach a contradiction and hence $v_{\infty}$ can only be a regular solution of (3.4). Yet this is impossible by (iv) of Theorem 3.9 and the reasoning as above.

As a consequence of Theorem 3.10 and Proposition 3.8, we have the following result which is in a direction opposite to that of Theorem 3.9.

Theorem 3.11. Suppose that $p>(n+l) /(n-2)$, and that the growth condition on $\phi$ in (i) of Theorem 3.10 holds. Then the conclusion of (i) in Theorem 3.10 holds true provided that

(i) when $(n+l) /(n-2)<p<(n+2+2 l) /(n-2), \phi \geq \underline{u}_{R}$ for some $R>0$; 
(ii) when $p=(n+2+2 l) /(n-2), \phi \geq \underline{u}_{\lambda}^{E}$ for some $\lambda>0$;

(iii) when $p>(n+2+2 l) /(n-2), \phi \geq \hat{u}_{\alpha}$ for some $\alpha>0$;

(iv) when $p>(n+2+2 l) /(n-2), \phi \geq \beta u_{\alpha}$ for some $\beta>1$ and some $\alpha>0 ;$ or when $p=(n+2+2 l) /(n-2), \phi \geq \beta u_{\lambda}^{E}$ for some $\beta>1$ and some $\lambda>0$

(v) when $p \geq p_{c}, \liminf _{|x| \rightarrow+\infty}|x|^{(2+l) /(p-1)} \phi(x)>L$.

Proof. (i)-(iv) are immediate from Theorem 3.10 and Proposition 3.8. To prove (v), we first assume that $\phi>0$. By (iv), it suffices to find $\beta>1$ and $\alpha>0$ such that $\phi \geq \beta u_{\alpha}$. Since $\liminf _{|x| \rightarrow+\infty}|x|^{(2+l) /(p-1)} \phi(x)>L=$ $\lim _{r \rightarrow+\infty} r^{(2+l) /(p-1)} u_{1}(r)$ (see (iv) of Proposition 3.4), there exist $R>0$ and $\beta>1$ such that $\phi(x) \geq \beta u_{1}(x)$ if $|x| \geq R$. By the fact that $\phi>0$ and $u_{\alpha}(r)=\alpha^{(2+l) /(p-1)} u_{1}(\alpha r) \leq \alpha^{(2+l) /(p-1)}$, there exists $1>\alpha>0$ such that $\phi(x) \geq \beta u_{\alpha}(x)$ if $|x| \leq R$. Since $u_{\alpha}$ is increasing in $\alpha$ (see (iv) of Proposition 3.7), we then have $\phi \geq \beta u_{\alpha}$ on $R^{n}$.

Now, suppose we only have $\phi \geq 0$. By Lemma 1.5 , for $0<t<T_{\phi}$,

$$
u(\cdot, t) \geq e^{t \Delta} \phi>0 \text {. }
$$

On the other hand, by the assumption, it is easily seen that

$$
\liminf _{|x| \rightarrow+\infty}|x|^{(2+l) /(p-1)}\left(e^{t \Delta}\right)(x)>L .
$$

Thus if we think of $u\left(\cdot, t_{0}\right)$ (for a positive $t_{0}<T_{\phi}$ ) as initial value, then the result for positive initial value proved above implies the desired result.

\section{Decay RATE}

In this section, we obtain the decay rate for global solutions of $(3.1)$ in some special cases.

Theorem 4.1. Suppose in (3.1), $l=0$ and $p>n /(n-2)$. Assume $\psi$ is a bounded nonnegative c.w. upper solution of (3.4) with $l=0$. If the initial value $\phi \leq \lambda \psi$ for some $0<\lambda<1$, then (3.1) has a unique global classical solution $u$ satisfying $0 \leq u \leq \lambda \psi$ and for $t>0$

$$
\|u(\cdot, t)\|_{L^{\infty}\left(R^{n}\right)} \leq\left(\left(\lambda^{1-p}-1\right)(p-1)\right)^{-1 /(p-1)} t^{-1 /(p-1)} .
$$

Remark. By Proposition 3.6 , if $\psi$ is radial, then necessarily $\psi$ is bounded.

Proof of Theorem 4.1. The global existence follows from Lemma 1.2 with $\bar{u}=$ $\lambda \psi$ and $\underline{u}=0(\lambda \psi$ is a c.w. upper solution of (3.1)). The uniqueness is immediate from Lemma 1.3. To prove the large time behavior of $u$, it suffices to take $\phi=\lambda \psi$. First, we assume $\psi$ is $C^{\infty}$ smooth, then $u$ is $C^{\infty}$ smooth to the boundary $t=0$. Consider $v=u_{t}+\delta u^{p}$ (a function similar to this was used in [10] in which the finite time blow-up was studied) where constant $\delta>0$ is to be determined later. By a straightforward computation we have

$$
v_{t}-\Delta v \leq p u^{p-1} v \text { on } R^{n} \times[0,+\infty) .
$$

Observe that

$$
\begin{aligned}
\left.v\right|_{t=0} & =\left.\left(u_{t}+\delta u^{p}\right)\right|_{t=0}=\left.\left(\Delta u+(1+\delta) u^{p}\right)\right|_{t=0} \\
& =\lambda \Delta \psi+(1+\delta) \lambda^{p} \psi^{p} \leq \lambda \psi^{p}\left((1+\delta) \lambda^{p-1}-1\right) \\
& =0 \quad \text { if } \delta=\lambda^{1-p}-1 .
\end{aligned}
$$


From Lemma 2.6, $u_{t} \leq 0$. So $v \leq \delta u^{p} \leq \delta \psi^{p}$. In particular, $v$ and $p u^{p-1}$ are bounded above. Thus by the Phragmèn-Lindelöf principle (see Lemma 1.3), $v \leq 0$, i.e., $u_{t}+\delta u^{p} \leq 0$ with $\delta=\lambda^{1-p}-1$. This in turn implies that

$$
u(x, t) \leq\left(\left(\lambda^{1-p}-1\right)(p-1)\right)^{-1 /(p-1)} t^{-1 /(p-1)} \text { for } t>0
$$

and this completes the argument for regular $\psi$.

For the general case, consider the bounded global classical solution $u_{\psi}$ of (3.1) with $l=0, \phi=\psi\left(u_{\psi}\right.$ is assured by Lemma 1.2 again). By uniqueness, this $u_{\psi}$ is the same one as in Theorem 2.5. Hence by the proof of Theorem 2.5, $u_{\psi}(\cdot, t) \rightarrow \psi(\cdot)$ in $L^{\infty}\left(R^{n}\right)$ as $t \rightarrow 0^{+}$. Also, by Lemma 2.6, $\partial u_{\psi} / \partial t \leq 0$ for $t>0$ and hence $\psi_{\varepsilon}(\cdot)=u_{\psi}(\cdot, \varepsilon)$ is a bounded smooth (by regularity theory) upper solution of (3.4) with $l=0$. Therefore, the conclusion for smooth $\psi$ implies that

$$
u_{\varepsilon}(x, t) \leq\left(\left(\lambda^{1-p}-1\right)(p-1)\right)^{-1 /(p-1)} t^{-1 /(p-1)}
$$

where $u_{\varepsilon}$ is the global classical solution of (3.1) with $l=0$ and $\phi=\lambda \psi_{\varepsilon}$. We claim that $u_{\varepsilon} \rightarrow u$ pointwise on $R^{n} \times[0,+\infty)$ (hence we are done). In fact, this follows from the continuity of solutions with respect to the initial value. This continuity can be proved by the integral equation and Gronwall inequality.

Corollary 4.2. Suppose in (3.1) that $p>n /(n-2)$ and $l=0$. If the initial value $\phi \leq \lambda u_{\text {s }}$ for some constant $\lambda<1$, then (3.1) has a unique global classical solution $u$ satisfying $u \leq \lambda u_{s}$ and

$$
\|u(\cdot, t)\|_{L^{\infty}\left(R^{n}\right)} \leq\left(\left(\lambda^{1-p}-1\right)(p-1)\right)^{-1 /(p-1)} t^{-1 /(p-1)} .
$$

Proof. Again, the uniqueness immediately follows from the Phragmèn-Lindelöf principle (see Lemma 1.3). On the other hand, exactly as in the proof of (iii) of Theorem 3.9, we can find a c.w. upper solution $\psi$ of (3.4) such that $\phi \leq \lambda \psi \leq$ $\lambda u_{s}$ when $n /(n-1)<p<p_{c}$, and $\phi \leq \lambda^{\prime} \psi \leq \lambda^{\prime} u_{s}$ when $p \geq p_{c}$, where $\lambda^{\prime}$ and $\psi$ can be chosen so that $\lambda^{\prime}$ can be arbitrarily close to $\lambda$ and $\lambda<\lambda^{\prime}<1$. By Theorem 4.1, in any case, (3.1) has a unique bounded global classical solution $u$ so that $0 \leq u \leq \lambda^{\prime} \psi\left(\leq \lambda^{\prime} u_{s}\right)$ and

$$
\|u(\cdot, t)\|_{\infty} \leq\left(\left(\left(\lambda^{\prime}\right)^{1-p}-1\right)(p-1)\right)^{-1 /(p-1)} t^{-1 /(p-1)} .
$$

Letting $\lambda^{\prime} \rightarrow \lambda$, we are done.

For (3.1) with $l \neq 0$, we are unable to obtain the decay rate for $\lambda$ close to 1 . However, we can do so for small $\lambda$ and large $p$. We shall use a self-similar solution, i.e., a solution $v$ of $v_{t}=\Delta v+|x|^{l} v^{p}$ with $v(x, t)=t^{-(2+l) / 2(p-1)} w(x / \sqrt{t})$, as an upper solution of (3.1). If $w$ is bounded, then we obtain a decay rate for the global solution of (3.1) (this idea was used in [24]). To find such a $v$, all we have to do is to find a radial $w>0$. By direct calculation, $w=w(r)$ should satisfy

$$
w^{\prime \prime}+\left(\frac{n-1}{r}+\frac{r}{2}\right) w^{\prime}+\frac{k}{2} w+r^{l} w^{p}=0, \quad r>0,
$$

where $k=(2+l) /(p-1), l>-2, n \geq 3$, and $p>1$. (4.1) with $l=0$ has been studied at least by [18] and [31]. We shall combine the ideas in these two papers to obtain global existence for (4.1). 
First, for local existence, following [18], we consider

$$
w(r)=w_{0}-\int_{0}^{r}\left(s^{n-1} e^{s^{2} / 4}\right)^{-1} d s \int_{0}^{s}\left(\frac{k}{2} w(t)+t^{l} w^{p}(t)\right) t^{n-1} e^{t^{2} / 4} d t,
$$

where $r \geq 0, w_{0}>0$, and $w>0$ on $[0,+\infty)$. Observe that a continuous solution $w$ of (4.2) is also a solution of (4.1) and that a solution of (4.1) which is continuous at $r=0$ is a solution of (4.2). On the other hand, by the standard contraction mapping argument, (4.2) has a unique positive continuous local solution $w$. To prove $w$ is global and positive, we observe from (4.2) that except at $r=0, w^{\prime}(r)<0$ before $w=0$ occurs, and hence it suffices to show that $w$ never vanishes. To this end, we shall use a Pohozaev-type identity which is directly inspired by the one in [31].

Lemma 4.3. If $w$ is a solution of (4.1) and $w$ is continuous at $r=0$, then for every $R>0$,

$$
\begin{gathered}
\int_{0}^{R}\left[\left(\frac{k}{2}-\frac{n}{4}\right) w^{2}+\left(\frac{n+l}{p+1}-\frac{n-2}{2}\right) r^{l} u^{p+1}-\frac{1}{2}\left(\frac{n}{2} w+r w_{r}\right)^{2}\right] r^{n-1} d r \\
=R^{n-1}\left[\left(\frac{k}{4}-\frac{n+2}{8}\right) R w^{2}(R)+\frac{n-2}{2} w(R) w_{r}(R)\right. \\
\left.+\frac{R^{2}}{2} w_{r}^{2}(R)+\frac{R^{l+1} w^{p+1}(R)}{p+1}\right] .
\end{gathered}
$$

Proof. By a straightforward calculation, for $r>0$ we have

$$
\begin{aligned}
& \frac{d}{d r}\left[r^{n-1}\left(-\frac{n+2}{8} r w^{2}+\frac{n-2}{2} w w_{r}+\frac{r^{2}}{2} w_{r}^{2}+r\left(\frac{k}{4} w^{2}+\frac{r^{l} w^{p+1}}{p+1}\right)\right)\right] \\
& =r^{n-1}\left[\left(\frac{k}{2}-\frac{n}{4}\right) w^{2}+\left(\frac{n+l}{p+1}-\frac{n-1}{2}\right) r^{l} w^{p+1}-\frac{1}{2}\left(\frac{n}{2} w+r w_{r}\right)^{2}\right]
\end{aligned}
$$

Integrating the above equation on $[\delta, R]$ and letting $\delta \rightarrow 0^{+}$, we obtain (4.3) by the fact that $r u_{r} \rightarrow 0$ as $r \rightarrow 0^{+}$, which is easily seen after differentiating (4.2).

Now we continue our discussion concerning the global existence of (4.1) (or (4.2)). Suppose that $R>0$ is the first zero of the local solution $w$. Then by the uniqueness for O.D.E., $w_{r}^{\prime}(R) \neq 0$. Therefore if in (4.3) $k / 2-n / 4 \leq 0$ and $(n+l) /(p+1)-(n-2) / 2 \leq 0$, i.e., if $p \geq \max \{(n+4+2) / n,(n+2+2 l) /(n-2)\}$, the left-hand side of $(4.3) \leq 0$ while the right-hand side $>0$. Thus

Lemma 4.4. When $p \geq \max \{(n+4+2 l) / n,(n+2+2 l) /(n-2)\},(4.1)$ has a global positive solution $w$ which is also continuous at $r=0$ and is decreasing in $r$.

Remark. In [18], Haraux and Weissler proved such a result when $l=0$ and even when $(n+2) / n<p<(n+2) /(n-2)$ if $w(0)$ is small.

Next, we prove $\lim _{r \rightarrow+\infty} r^{k} w(r)$ exists and is positive (this is also proved in [18] when $l=0)$. We divide the proof into several lemmas below.

Lemma 4.5. $r^{k} w(r)$ is bounded. 
Proof. Our approach is different from that of [18]. Let $f(t)=r^{k} w(r), t=$ $\log r$, then $f>0, \lim _{t \rightarrow-\infty} f(t)=0$ and

$$
f^{\prime \prime}+\left(c_{0}+e^{2 t} / 2\right) f^{\prime}+f^{p}-L^{p-1} f=0 \text { on }(-\infty,+\infty),
$$

where

$$
c_{0}=\frac{n-2}{p-1}\left(p-\frac{n+2+2 l}{n-2}\right) \geq 0
$$

and $L$ is as in (0.7). Suppose $r^{k} w(r)$ is unbounded, then it is easy to see from (4.4) that either $f$ is increasing for large $t$ or $f$ oscillates around $L$. If $f$ is increasing for large $t$, then there exists a $c>0$ such that $f^{p}-L^{p-1} f \geq c f$ for a constant $c>0$ and for large $t$. Then (4.4) implies that $f^{\prime \prime}+c f \leq 0$ for large $t,\left(f^{\prime}\right)^{2}+c f^{2}$ is therefore nonincreasing for large $t$. This contradicts the assumption that $f$ is unbounded. If $f$ oscillates around $L$, choose $t_{k} \rightarrow+\infty$ such that $f$ achieves local maximum at each $t_{k}$ and $f\left(t_{k}\right) \rightarrow+\infty$. Multiplying (4.4) by $f^{\prime}$ and integrating on $\left[t_{1}, t_{k}\right]$, we have

$$
\left.\frac{1}{2}\left(f^{\prime}\right)^{2}\right|_{t_{1}} ^{t_{k}}+\int_{t_{1}}^{t_{k}}\left(c_{0}+\frac{e^{2 t}}{2}\right)\left(f^{\prime}\right)^{2}+\left.\left(\frac{1}{p+1} f^{p+1}-\frac{L^{p-1}}{2} f^{2}\right)\right|_{t_{1}} ^{t_{k}}=0 .
$$

So $\int^{\infty}\left(c_{0}+e^{2 t} / 2\right)\left(f^{\prime}\right)^{2}<+\infty$ and $f\left(t_{k}\right)$ is bounded which contradicts the assumption that $f\left(t_{k}\right) \rightarrow+\infty$.

Next, by some easy modifications of Proposition 3.2 and Proposition 3.4 in [18], we have the following three lemmas.

Lemma 4.6. If for some positive $\sigma \geq 1 /(p-1), r^{\sigma} w(r)$ is bounded, then $\left|r^{\sigma+1} w^{\prime}(r)\right|$ is also bounded at $r=+\infty$.

Lemma 4.7. $\lim _{r \rightarrow+\infty} r^{k} w(r)$ exists.

Lemma 4.8. If $\lim _{r \rightarrow+\infty} r^{k} w(r)=0$, then for all positive $m, \lim _{r \rightarrow+\infty} r^{m} w(r)$ $=\lim _{r \rightarrow+\infty} r^{m+1} w^{\prime}(r)=0$.

Now we can prove that the $w$ in Lemma 4.4 satisfies $\lim _{r \rightarrow+\infty} r^{k} w(r) \neq 0$. For otherwise, by using Lemma 4.7 and letting $R \rightarrow+\infty$ in (4.3), we have that the left-hand side of (4.3) is negative and the right-hand side is 0 .

Finally, we return to the decay rate problem for (3.1).

Theorem 4.9. Suppose that in (3.1), $p \geq \max \{(n+4+2 l) / n,(n+2+2 l) /(n-2)\}$. If the initial value $\phi(x) \leq \lambda(1+|x|)^{-(2+l) /(p-1)}$ on $R^{n}$ for some small $\lambda>0$, then (3.1) has a (unique) global solution $\left(C_{B}\right.$-mild when $-2<l<0$ and classical when $l \geq 0$ ) satisfying

Proof. Let

$$
\|u(\cdot, t)\|_{L^{\infty}\left(R^{n}\right)} \leq M t^{-(2+l) / 2(p-1)} \text { for } t>0 \text { and some } M>0 .
$$

$$
h(x, t)=v(x, t+1)=(t+1)^{-(2+l) / 2(p-1)} w\left(\frac{|x|}{\sqrt{t+1}}\right) \quad \text { for } t \geq 0 .
$$

Of course, when $l \geq 0, h$ is a classical solution of $u_{t}=\Delta u+|x|^{l} u^{p}$. When $l<0$, it is not hard to prove that $h$ is a c.w. solution of $u_{t}=\Delta u+|x|^{l} u^{p}$ 
and hence by the remark following Definition $2.1, h$ is also a $C_{B}$-mild solution (note $w$ is bounded and therefore $h$ is bounded). Observe

$$
\lim _{|x| \rightarrow+\infty}|x|^{(2+l) /(p-1)} h(x, 0)=\lim _{|x| \rightarrow+\infty}|x|^{(2+l) /(p-1)} w(|x|)>0
$$

by the result above. So if $\lambda$ is small and $\phi(x) \leq \lambda(1+|x|)^{-(2+l) /(p-1)}$, we have $\phi \leq\left. h\right|_{t=0}$. Then $h$ is an upper solution of (3.1). Applying Theorem 2.4 (when $-2<l<0$ ) and Lemma 1.2 (when $l \geq 0$ ), we conclude that $(3.1)$ has a global solution $u$ with $0 \leq u \leq h$. Since $w$ is bounded, our proof is now finished.

\section{A REMARK ON THE FIRST BOUNDARY VALUE PROBLEM}

Consider the first initial-boundary problem

$$
\begin{aligned}
& u_{t}=\Delta u+|x|^{l} u^{p}, \quad x \in D, t>0, u \geq 0, \\
& \left.u\right|_{s}=0, \\
& \left.u\right|_{t=0}=\phi \in C_{0}(\bar{D})=\left\{\phi \in C(\bar{D})|\phi|_{\partial D}=0\right\}, \quad \phi \not \equiv 0, \phi \geq 0,
\end{aligned}
$$

where $D$ is a domain in $R^{n}$ with $\partial D$ satisfying the exterior sphere condition, $S=\partial D \times(0,+\infty), p>1, l>-2$, and $n \geq 3$.

As for the Cauchy problem, when $0 \in \bar{D}$ and $l<0$, it is necessary and convenient to introduce the concept of "mild" solution for (5.1). This can be done by replacing $e^{t \Delta}$ in Definition 2.1 by the Green function $G$. Note by the maximum principle, $0<G<\Gamma$ ( $\Gamma$ is the standard heat kernel). Theorem 2.3 and Theorem 2.4 still hold for (5.1) with some obvious modifications, hence the local existence and comparison principle are valid for (5.1) even when $0 \in \bar{D}$ and $-2<l<0$. Observe that if we let the initial value $\phi=0$ outside $D$ (we still denote it by $\phi$ ), then the corresponding solution (if any) of the Cauchy problem (3.1) is an upper solution of (5.1). Therefore, by Theorem 2.4 (modified for (5.1)) and Lemma 1.2 with $\bar{u}=$ solution of (3.1) and $\underline{u}=0$, we have for the existence and large time behavior of global solution of (5.1) that Theorem 3.9, Corollary 4.2, and Theorem 4.9 with obvious modifications that for $(5.1)$.

\section{ACKNOWLEDGMENTS}

This paper comes from the author's thesis under the supervision of Professor Wei-Ming $\mathrm{Ni}$, to whom I wish to express my sincere gratitude for his constant encouragement, valuable suggestions and help. I am gratefully indebted to Professor Hans Weinberger for helpful conversations, to Professor Yi Li for a helpful comment on the original version of this paper, to Professor Steven Rosencrans and the referee for their generous help in the presentation of this paper.

\section{REFERENCES}

1. D. G. Aronson and H. F. Weinberger, Multidimensional nonlinear diffusion arising in population genetics, Adv. Math. 30 (1978), 33-76.

2. T. Aubin, Nonlinear analysis on manifolds. Monge-Ampére equations, Springer-Verlag, New York, Heidelberg, and Berlin, 1982. 
3. C. Bandle and H. A. Levine, On the existence and nonexistence of global solutions of reactiondiffusion equations in sectorial domains, Trans. Amer. Math. Soc. 316 (1989).

4. __ Fujita type results for convective like reaction diffusion equations in exterior domains, Z. Angew. Math. Phys. 40 (1989), 665-676.

5. L. Caffarelli, B. Gidas, and J. Spruck, Asymptotic symmetry and local behavior of semilinear elliptic equations with critical Sobolev growth, Comm. Pure Appl. Math. 42 (1989), 271-297.

6. R. Fowler, The solution of Emden's and similar differential equations, Monthly Notices Roy. Astronom. Soc. 91 (1930), 63-91.

7. __ Further studies of Emden's and similar differential equations, Quarterly J. Math. Oxford Ser. 2 (1931), 259-288.

8. A. Friedman, A strong maximum principle for weakly subparabolic functions, Pacific $\mathbf{J}$. Math. 11 (1961), 175-184.

9. __, Partial differential equations of parabolic type, Prentice-Hall, Englewood Cliffs, N. J., 1964.

10. A. Friedman and B. Mcleod, Blow-up of positive solutions of semilinear heat equations, Indiana Univ. Math. J. 34 (1985), 425-447.

11. H. Fujita, On the blowing up of solutions of the Cauchy problem for $u_{t}=\Delta u+u^{1+\alpha}$, J. Fac. Sci. Tokyo Sect. IA Math. 13 (1966), 109-124.

12. $\ldots$, On some nonexistence and nonuniqueness theorems for nonlinear parabolic equations, Proc. Sympos. Pure Math. vol. 18, part I, Amer. Math. Soc., Providence, R. I., 1968, pp. 138-161.

13. B. Gidas, W.-M. Ni, and L. Nirenberg, Symmetry and related properties via the maximum principle, Comm. Math. Phys. 68 (1979), 209-243.

14. _ Symmetry of positive solutions of nonlinear elliptic equations in $R^{n}$, Advances in Math. Suppl. Stud. 7A, Math. Anal. Appl., part A, 1981, pp. 369-402.

15. B. Gidas and J. Spruck, Global and local behavior of positive solutions of nonlinear elliptic equations, Comm. Pure Appl. Math. 23 (1981), 525-598.

16. D. Gilbarg and N. Trudinger, Elliptic partial differential equations of second order, SpringerVerlag, New York, Heidelberg, and Berlin, 1983.

17. J. Hale, Theory of functional differential equations, Springer-Verlag, New York, Heidelberg, and Berlin, 1977.

18. A. Haraux and F. B. Weissler, Non-uniqueness for a semilinear initial value problem, Indiana Univ. Math. J. 31 (1982), 167-189.

19. K. Hayakawa, On nonexistence of global solutions of some semilinear parabolic equations, Proc. Japan Acad. 49 (1973), 503-525.

20. D. Joseph and T. Lundgren, Quasilinear Dirichlet problems driven by positive sources, Arch. Rational Mech. Anal. 49 (1973), 241-269.

21. S. Kaplan, On the growth of solutions of quasilinear parabolic equations, Comm. Pure Appl. Math. 16 (1963), 305-333.

22. K. Kobayashi, T. Siaro, and H. Tanaka, On the blowing up problem for semilinear heat equations, J. Math. Soc. Japan 29 (1977), 407-424.

23. O. Ladyzenskaja, U. Solonikov, and N. Uralceva, Linear and quasilinear equations of parabolic type, Amer. Math. Soc., Providence, R. I., 1968.

24. T.-Y. Lee and W.-M. Ni, Global existence, large time behavior and life span of solutions of semilinear parabolic Cauchy problem, Trans. Amer. Math. Soc. 333 (1992), 365-371.

25 . $\mathrm{H}$. A. Levine, The long time behavior of solutions of reaction-diffusion equations in unbounded domains: a survey, Proc. 10th Dundee Conf. Theory of Ordinary and Partial Differential Equations, 1988.

26. _ . The role of critical exponents in blow up theorems, SIAM Rev. 32 (1990), 262-288.

27. W.-M. Ni, Uniqueness, nonuniqueness and related questions of nonlinear elliptic and parabolic equations, Proc. Sympos. Pure Math., vol. 45, part 2, Amer. Math. Soc., Providence, R. I., 1986, pp. 229-241. 
28. _. On the elliptic equation $\Delta u+K(x) u^{(n+2) /(n-2)}=0$, its generalizations, and applications in geometry, Indiana Univ. Math. J. 31 (1982), 493-529.

29. W.-M. Ni and J. Serrin, Topics in partial differential equations, Lecture Notes, Univ. of Minnesota, 1987-1988.

30. W.-M. Ni and S. Yotsutani, Semilinear elliptic equations of Matukuma-type and related topics, Japan J. Appl. Math. 5 (1988), 1-32.

31. L. A. Peletier, D. Terman, and F. B. Weissler, On the equation $\Delta u+\frac{1}{2} x \cdot \nabla u+f(u)=0$, Arch. Rational Mech. Anal. 94 (1986), 83-99.

32. M. H. Protter and H. F. Weinberger, Maximum principles in differential equations, PrenticeHall, Englewood Cliffs, N. J., 1967.

33. D. H. Sattinger, Monotone methods in nonlinear elliptic and parabolic boundary value problems, Indiana Univ. Math. J. 21 (1972), 979-1000.

34. F. B. Weissler, Local existence and nonexistence for semilinear parabolic equations in $L^{p}$, Indiana Univ. Math. J. 29 (1980), 79-101.

35. __ Existence and non-existence of global solutions for a semilinear heat equation, Israel J. Math. 38 (1981), 29-40.

36. Q.-X. Ye and Z-Y. Li, An introduction to reaction diffusion equations, Science Press, Beijing (in Chinese).

Department of Mathematics, Tulane University, New Orleans, Louisiana 70118

E-mail address: xdw@tulmath.math.tulane.edu 